This item was submitted to Loughborough's Research Repository by the author.

Items in Figshare are protected by copyright, with all rights reserved, unless otherwise indicated.

\title{
Surface forces action in a vicinity of three phase contact line and other current problems in kinetics of wetting and spreading
}

PLEASE CITE THE PUBLISHED VERSION

http://dx.doi.org/10.1016/j.cis.2010.02.002

PUBLISHER

(C) Elsevier

VERSION

AM (Accepted Manuscript)

LICENCE

CC BY-NC-ND 4.0

\section{REPOSITORY RECORD}

Starov, Victor. 2011. "Surface Forces Action in a Vicinity of Three Phase Contact Line and Other Current Problems in Kinetics of Wetting and Spreading". figshare. https://hdl.handle.net/2134/7672. 
This item was submitted to Loughborough's Institutional Repository (https://dspace.lboro.ac.uk/) by the author and is made available under the following Creative Commons Licence conditions.

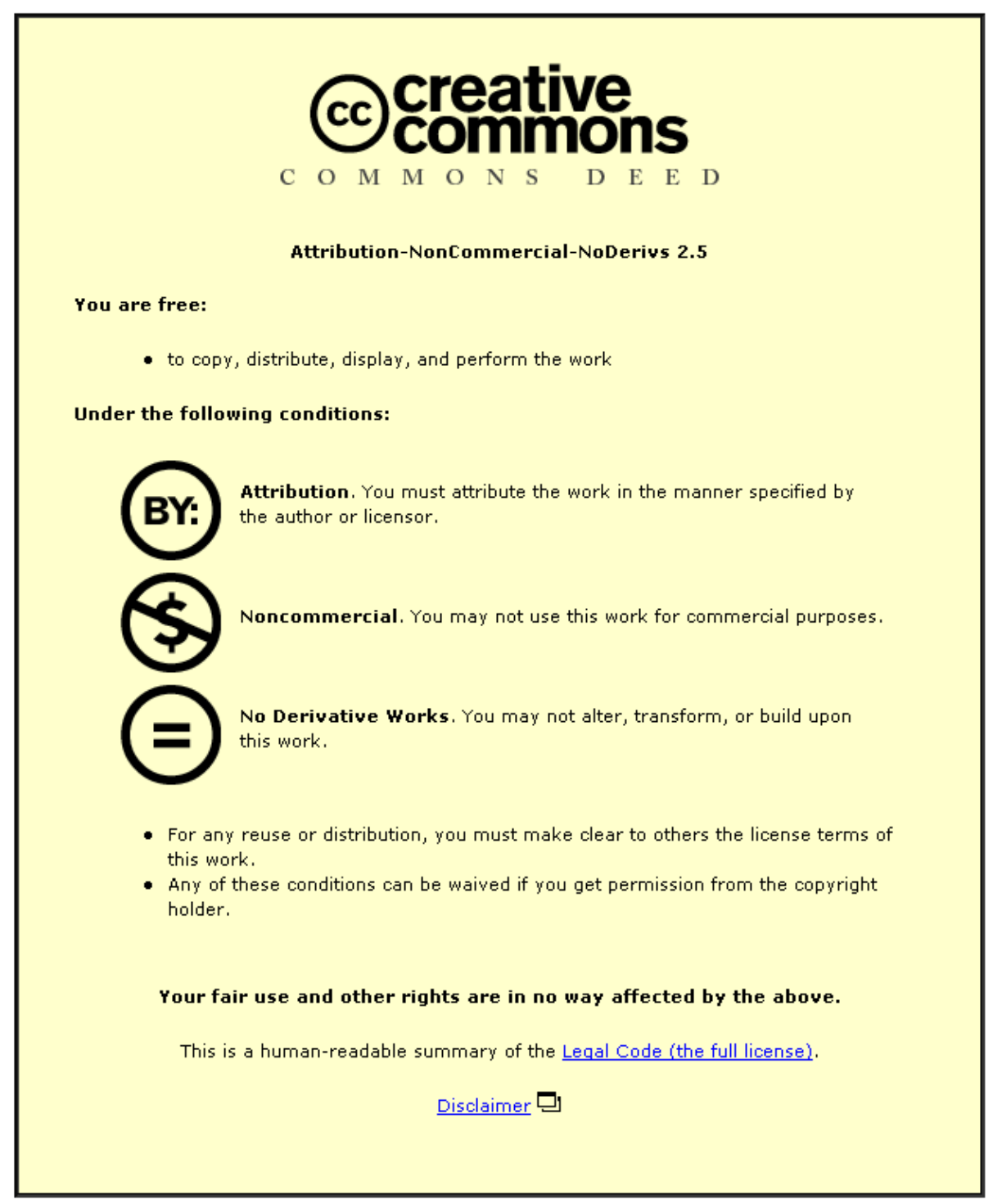

For the full text of this licence, please go to: http://creativecommons.org/licenses/by-nc-nd/2.5/ 


\section{Surface forces action in a vicinity of three phase contact line and other current problems in kinetics of wetting and spreading}

Victor M Starov, Department of Chemical Engineering, Loughborough University, Loughborough, LE11 3TU, UK, V.M.Starov@lboro.ac.uk

\section{Contents}

Abstract

Introduction

1. Wetting phenomena and surface forces

Derjaguin's pressure

Contact angle and adsorption on solid substrates

Equilibrium contact angle in the case of partial wetting

2. Hysteresis of contact angle on smooth homogeneous substrates

3. Kinetics of spreading and surface forces

4. Spreading over porous substrates

5. Simultaneous spreading and evaporation

6. Spreading of aqueous droplets and aqueous surfactant solutions

Spreading of aqueous drops induced by the overturning of amphiphilic molecules or their fragments in the surface layer of an initially hydrophobic substrate

Spreading of surfactants over thin liquid layers.

Acknowledgement

References 


\section{Abstract}

The most important problem in kinetics of wetting and spreading from the author's point of view is a consideration of combined surface forces and capillary action in a vicinity of the apparent three phase contact line. The latter is equally important at consideration of static or dynamics. Other current trends in kinetics of wetting and spreading are also briefly reviewed. It is impossible to cover the whole literature on the subject: it was around 5,000 publications on that subject in 2009 only and the total number of publication in the area is 65, 917 (according to Science Direct). The problems to be solved in the area are marked in italic bold and underlined.

\section{Introduction}

Wetting and spreading phenomena are used by human beings from the very beginning of our history. It is a reason why those phenomena attract attention of scientists for ages and as it is shown below they still do (see Fig. 1).

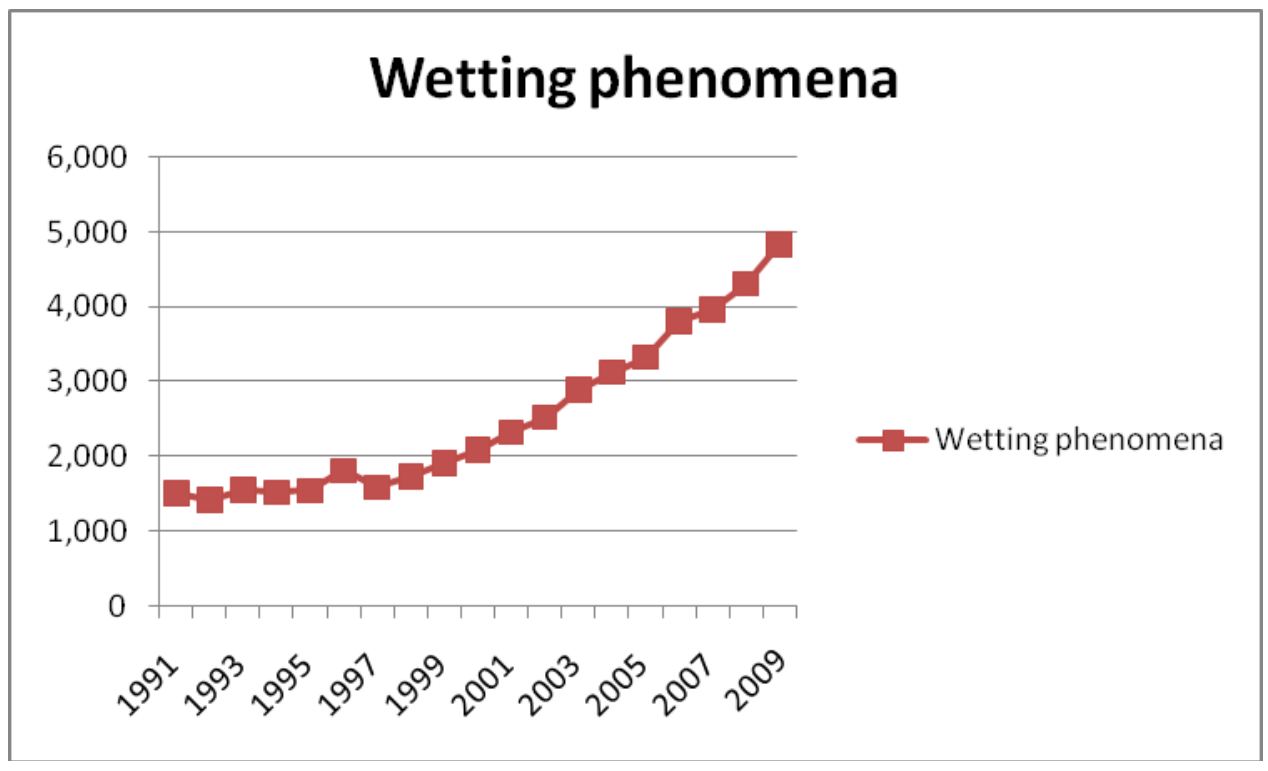

Fig. 1. Number of publications since 1991 on wetting phenomena. Source: Science Direct. Total number of publications in the area 65,917.

Fig. 1 clearly shows that spreading/wetting phenomena still attract a lot of attention from both scientists and industrialists. In spite of such considerable interest in the area of wetting/spreading there is no specific EU or international conference on "Spreading and wetting phenomena" (compare with the situation in nanotechnology, for example). 
Fig. 1 shows that there was more than 65 thousand publications on spreading and wetting The huge number of publications in the area means that it is impossible to review all those publications.

It is the reason why below the author concentrats mostly on the most important problem in the area of spreading/wetting: a consideration of a combined action of surface and capillary forces in a vicinity of an apparent three phase contact line, and on other basic problems whithout those the further progress in the area is impossible according to the author's opinion.

Why do droplets of different liquids deposited on the identical solid substrate behave so differently? Why identical droplets, for example, aqueous droplets, deposited on different substrates behave also differently?

A mercury droplet does not spread on a glass substrate. It rather forms a spherical cap with the contact angle bigger than $\pi / 2$ (Fig. 1a). An aqueous droplet deposited on the identical glass substrate spreads only partially down to some contact angle, $\theta$, which is in between 0 and $\pi / 2$ (Fig. 1b). However, an oil droplet (hexane or decane) deposited on the same glass substrate spreads out completely (Fig.1c), and the contact angle decreases with time down to the zero value.

By convention, the contact angle is measured inside the liquid phase Figs. 1a, 1b, 1c).

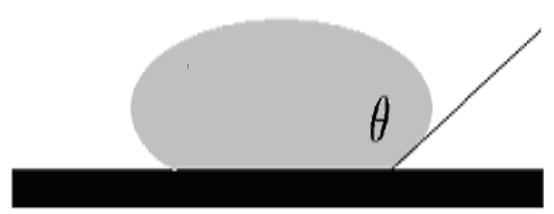

Fig.1a. Non-wetting case: contact angle is bigger than $\pi / 2$. Examples: a mercury droplet on a glass surface, or a water droplet on Teflon surface. 


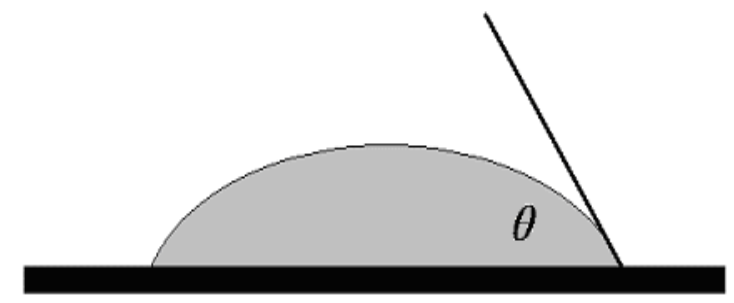

Fig.1b. Partial wetting case: the contact angle is in between 0 and $\pi / 2$. Examples: a water droplet on a glass, mica, silicon wafer surfaces.

These three cases (Figs. 1a, 1b, and 1c) are referred to as: non-wetting, partial wetting and complete wetting, respectively. In all three cases mentioned above a three-phase contact line (solid-liquid-vapour) is formed by a liquid drop deposited on a solid surface.

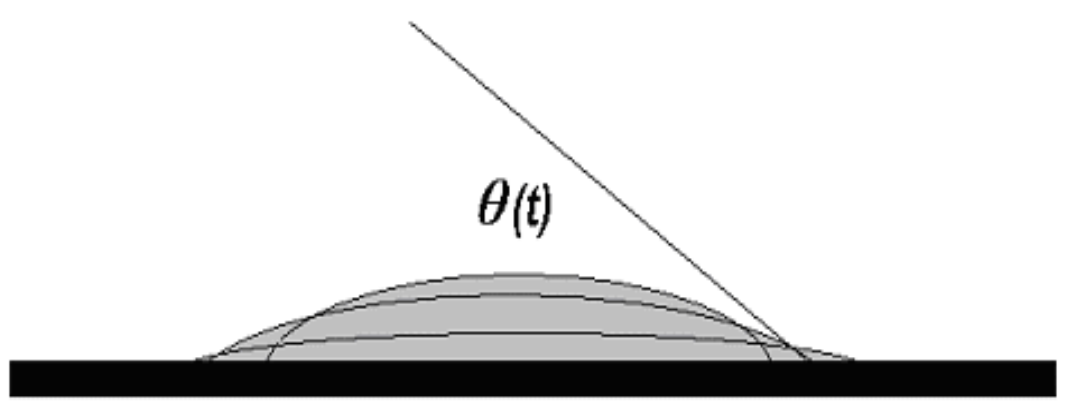

Fig.1c. Complete wetting case: the droplet spreads out completely and the dynamic contact angle tends to zero with time. Example: an oil droplet on a glass surface.

Needless to say, wettability (complete, partial or none) is determined by the nature of both the liquid and the solid substrate and their interactions. 
Below we consider behaviour of two the most important liquids in contact with solid substrates as an example: water or aqueous solutions (polar liquids) and oils (non polar liquids).

Consideration of equilibrium wetting phenomena is frequently based on well known Young's equation [1] and its modifications for rough surfaces [2, 3]. There are numerous derivations of Young's equation, which can be roughly subdivided into two big groups: thermodynamic derivations, and mechanical derivations. The most important assumption of all those derivations is as follows: the drop retains a spherical shape up to the contact with a solid substrate that is up to the three phase contact line where the thickness of the liquid becomes zero. Below we show that this assumption is in a contradiction with the scientific development in the area.

Starting from the beginning of $20^{\text {th }}$ century new phenomena, which take place in thin liquid films and layers has been under scrutiny of a wide range of scientists ever since: those phenomena are now refereed to as "surface phenomena" or "surface forces" [4-7]. Surface forces are briefly reviewed in Section 1. Manifestation of the surface forces action is the appearance of the disjoining pressure [4] in thin liquid films and layers. It is necessary to note that the latter name "disjoining pressure" is a very misleading one: that pressure could be either disjoining or conjoining. However, because of historical reasons the term "disjoining pressure" is widely used. Note, it was suggested in [8] to rename "disjoining pressure" and to use "Derjaguin's pressure" instead to avoid the misunderstanding and to recognise the contribution of Derjaguin to the area. It is up to the scientific community to decide if a new name is appropriate. Below we use "Derjaguin's pressure" instead of "disjoining pressure".

Surface forces come into play in a vicinity of a tree phase contact line, where the liquid thickness becomes smaller than the range of action of surface forces. The latter range is usually around $100 \mathrm{~nm}$. The action of surface forces results in a substantial deviation of a liquid profile from a spherical shape. The latter requires a revision of Young equation and the latter is undertaken in Section 1. The revised theory results in a completely new approach to the equilibrium contact angle [9].

Consideration in Section 1 shows that all equilibrium phenomena in the case of both complete and partial wetting can be described based on a consideration 
of combined action of capillary and surface forces action in a vicinity of a three phase contact line [9].

However, the situation is far from being understood in the case nonwetting: it is still a challenge to understand surface forces acting in a vicinity of an apparent three phase contact line and wetting phenomena in this case.

Based on a consideration of surface forces the kinetics of spreading can be equally investigated. The latter has been done in the case of complete wetting and this approach is to be extended to the case of partial wetting (Section 2) [8, 9].

Note, the kinetics of spreading in the case partial wetting has not been finalized yet based on the previous approach. There is a good reason for that: this reason is a presence of hysteresis of contact angle on smooth homogeneous substrates. The latter is determined by the s-shape of the Derjaguin's pressure in the case of partial wetting. Note, the consideration of hysteresis of contact angle based of consideration of combined action of both capillary and surface forces on rough surfaces to the best of the author's knowledge has never been undertaken before and it is the future challenge.

The discussion below shows that both equilibrium and kinetic wetting phenomena can be described based on a consideration of combined capillary and surface force action in a vicinity of a three phase contact line. There are a number of unsolved problem in this direction. However, the author believes that this is the main stream direction of science in the area of wetting and spreading. That is a considerable part of the paper below is devoted to the consideration of a combined capillary and surface forces action in a vicinity of the three phase contact line in the case of both equilibrium and dynamics.

Kinetics of spreading of aqueous surfactant solutions over hydrophobic substrates is one of the most important subjects in the area of wetting and spreading. Pure aqueous droplets do not wet hydrophobic substrate (that is, the contact angle is bigger than $\pi / 2$ ). However, if after (or before the deposition) surfactants are added to the aqueous droplet, the latter starts spreading over the hydrophobic substrate. Scientists accumulated a considerable amount of information/experience in the area of spreading of surfactant solutions over 
hydrophobic substrates. As it was already mentioned above in a vicinity of an apparent three phase contact line surface forces unavoidable come into play. The surface forces action in the presence of surfactants is more complicated than in a pure liquids: surface forces are modified by the presence of surfactants, adsorption at all three interfaces (liquid-air, liquid-solid and solid-vapour) takes place and the latter may results in Marangoni phenomenon, which make the consideration even more complicated. Do we understand from this point of view why adding of surfactants to aqueous solutions results in a spreading of surfactant solutions over hydrophobic substrate? According to the author's opinion the answer is "no". To the best of the author's knowledge the surface forces action in the presence of surfactants in a vicinity of the apparent three phase contact line on hydrophobic substrates has not been understood yet. That is, in this area the scientists are forced to use Young's equation in spite of our firm understanding that this equation is in a contradiction with a modern science. However, it is necessary to keep in mind that conclusion made based on Young's equation must be validated against experimental data, without such validation any conclusion cannot be seriously considered: Young's equation ignores the surface forces action in a vicinity of the three phase contact line, which is in a contradiction with the modern science.

A similar situation takes place at the consideration of kinetics of spreading and simultaneous evaporation. The influence of surface forces in the course of those two interconnected processes was well recognised (see [10] and the references there in). More recently it has been proven experimentally that the rate of evaporation is proportional to its perimeter [11]. Note, according to that conclusion the evaporation proceeds mostly in a vicinity of the three phase contact line, that is, precisely in the region there surface forces are the most important. The first attempt to give a physical explanation of the peculiar behaviour of the evaporation flux was given in [12] in the case of a steady state evaporation from the meniscus. For some reason the theory presented in [12] is not widely known in the community involved in the area. Situation is more complicated in the case of spreading droplets. Influence of surface forces on kinetics of simultaneous spreading and evaporation of droplet is to be investigated.

In the case of spreading and evaporation of surfactant solutions the situation is even more complicated: the influence of surface forces and 
surfactants overlaps. Experimental investigations in this area started only recently [13].

Spreading over porous substrates is an interesting and challenging problem [14]. In this case of spreading over thin porous layers the situation is clarified recently (complete wetting case). However, the spreading over "thick porous" substrates is still a challenge even in the case of complete wetting. The situation is even more complicated in the case of spreading of surfactant solutions over porous substrates in the cases of partially wetting [14] or non-wetting and investigations here to be carried out.

From other problems, which are not considered below the author would like to mention two challenging problems: spreading of proteins over both hydrophobic and hydrophilic surfaces and spreading of nano-suspensions (nanofluids), which can form structures in a vicinity of the moving three phase contact line [15]. The spreading of protein solutions over various substrates is of a substantial industrial importance. However, surprisingly those processes are very poorly investigated: there are only scattered publications in the area. The spreading of nanofluids is definitely a new direction and has potentially a wide range of applications.

\section{Wetting phenomena and surface forces}

Derjaguin's pressure

It was mentioned in the Introduction that the surface forces (that is forces acting in thin liquid films/layer) were discovered in the beginning of the $20^{\text {th }}$ century and it resulted in a breakthrough in understanding of stability and coagulation of colloidal suspensions/emulsions. It is well established that the range of surface forces action is around $100 \mathrm{~nm}$. The components of those surface forces are: molecular (or dispersion, or Van der Waals) component, electrostatic component caused by an overlapping of electrical double layers of neighbouring charged surfaces, structural component caused by water dipoles orientation in a vicinity of surfaces of neighbouring surfaces and overlapping of those oriented layers, steric caused by overlapping adsorbed polymer/protein/surfactant layers and other components $[4,5,6,9]$. 
In the case of a liquid droplet on a solid substrate or a liquid inside a capillary (a simple model of a porous medium) in a vicinity of the three phase contact line the liquid thickness tends to zero and becomes less than $100 \mathrm{~nm}$ and, hence, fells into the realm of surface forces action. Hence, the liquid layers in a vicinity of the three phase contact line are in the range of the same surface forces as in the case of colloidal particles. A very important observation is as follow, the surface forces in thin liquid films in a vicinity of a three phase contact line include contributions of all the same components as in the case of interaction of colloidal particles: van der Waals, electrostatic, steric, interaction caused by the orientation of water dipoles at both water-solid and water-vapour interfaces. However, there is one very important difference in the case of droplets/menisci: interaction between identical colloidal particles or droplets is symmetrical, but the surface forces in thin liquid films in a vicinity of the three phase contact line are highly asymmetrical because two different interfaces are involved: a liquid-solid and a liquid-vapour. The latter feature determines the specific properties of wetting/spreading phenomena and probably explains why wetting/spreading phenomena based on the consideration of colloidal forces have been much less investigated than the interaction between colloidal particles.

It has been adopted now that the total Derjaguin's pressure, $\Pi(h)$, where $h$ is the film thickness, is a sum of mentioned above components $\Pi(h)=\Pi_{m}+\Pi_{e}+\Pi_{s}+\ldots$, where

- molecular or Van der Waals component, $\Pi_{m}$, is determined by the fluctuating electric field created by liquid molecules. There is a well established expression for this component $\Pi_{m}=\frac{A}{h^{3}}$, where $A$ is the Hamaker constant. If $A>0$ and that components is the only one acting in thin liquid layer, then the latter is the complete wetting case only: oils on glass, mica, metal and other surfaces. If $A<0$ and that components is the only one acting in thin liquid layer, then the latter is the complete non-wetting case only (that is, the contact angle is equal to $180^{\circ}$ ). The complete non-wetting has never realised in nature. Note, there is a tendency in the literature to exaggerate the importance of the latter component and to use 
that component only in the case of water and aqueous solutions. The latter is completely wrong as we see below;

- electrostatic component, determined by an overlapping of electrical double layers in a vicinity of charged liquid-vapour and liquid-solid interfaces, $\Pi_{e}$;

- structural component, determined by water dipoles orientation in a vicinity of liquid-vapour and liquid-solid interfaces. It comes into play then those layers overlap, $\Pi_{s}$.

As a result the Derjaguin's pressure isotherm (measured at constant temperature) has the following peculiar s-shaped form (Fig.2).

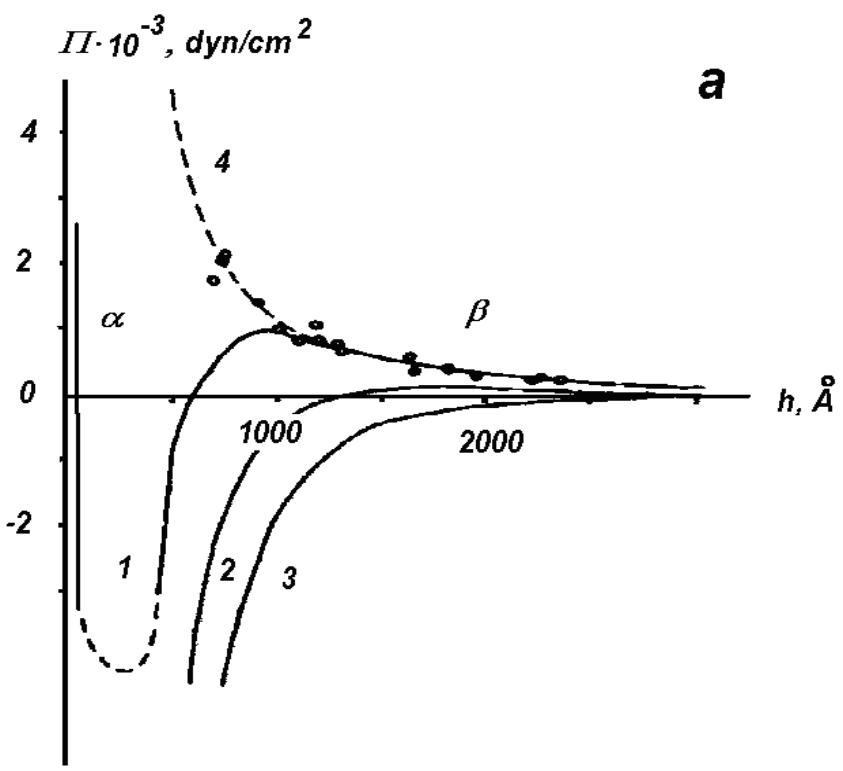




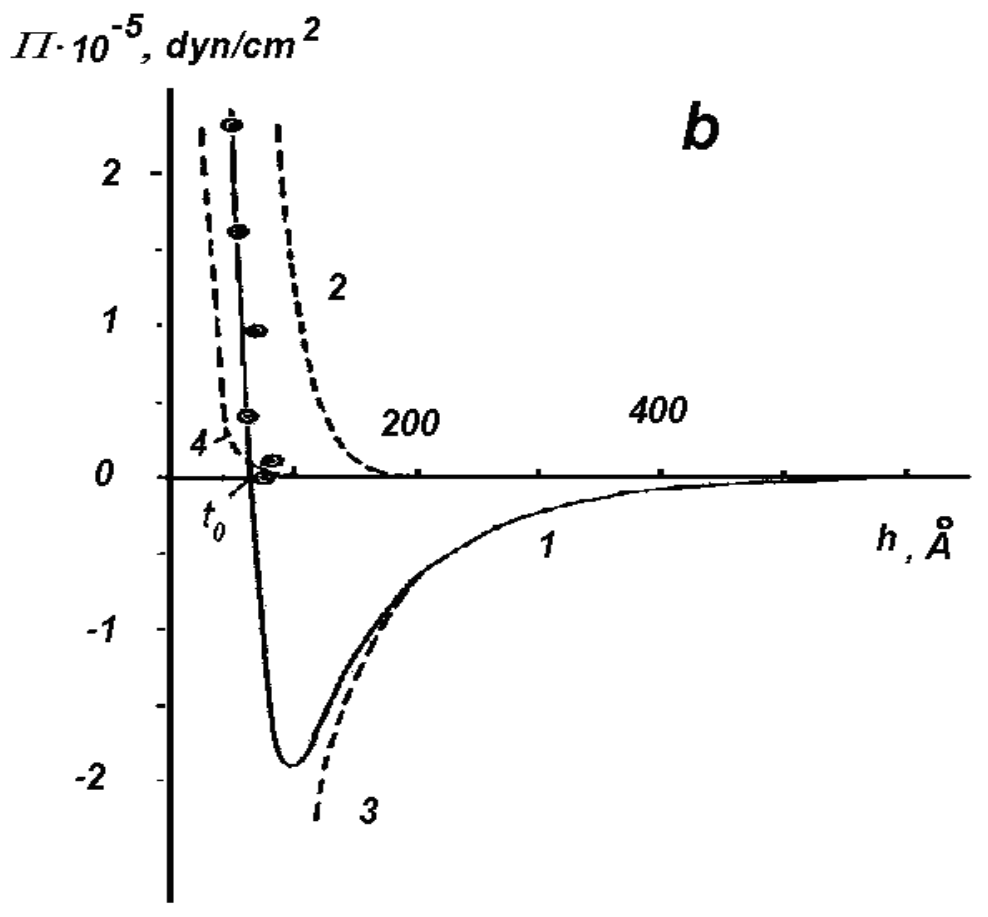

Fig. 2. Calculated and experimentally measured isotherms of Derjaguin's pressure, $\Pi(h)$, of water films on the quartz surface at concentration of $\mathrm{KCl} \mathrm{C}=10^{-5}$ mole/l, $\mathrm{pH}=7$, and dimensionless $\varsigma$ potential of the quartz surface equals to -6 .

(a) Within the region of large thicknesses: dimensionless $\varsigma$ potential of the film-air interface equals to -2.2 (curve 1), -1 (curve 2), and 0 (curve 3); (b) within the region of small thicknesses: dimensionless $\varsigma$ potential of the film-air interface equals to -2.2

(curve 1). The structural component, $\Pi_{s}(h)$, of the Derjaguin's pressure isotherm and electrostatic component, $\Pi_{e}(h)$, are shown by curves 2 and 3 , respectively. Note the difference in scales of Derjaguin's pressure in Figs 2a and 2b. Redrawn from [4].

The Derjaguin's pressure isotherms measured and/or calculated for flat liquid films are used for calculations of liquid profiles in a vicinity of a three phase contact line. The latter is acceptable only if the liquid profile has a low slope. That is, $\underline{\mathbf{a}}$ theory of Deriaguin's pressure in the case of moderately sloped liquid profiles is a challenge. Note, even in a relatively simple case of molecular component only (that is, complete wetting case) an attempt to calculate the Derjaguin's pressure in the case of moderate slopes resulted in a prediction of a contradictory equilibrium contact angle different from zero [16]. The latter is in a contradiction with current 
understanding of surface forces (see below). There is no mistake in calculations presented in ref [16]: the latter is a result of adopted in [16] direct integration (summation) of forces between molecules. Unfortunately those forces are nonadditive nature and the direct summation results in erroneous conclusions.

Fig. 2 shows that until now the Derjaguin's pressure has been measured in the region, which corresponds to case of undersaturation only. It is caused by the experimental method used for those measurements [17]. Measurements of Derjaguin's pressure in the region, which corresponds to the oversaturation is a challenge: a new experimental method is to be developed for those measurements.

Contact angle and adsorption on solid substrates

Before going further we consider a consequence of vapour adsorption on solid substrates.

It is well known from the theory of adsorption that vapours adsorb on solid substrates. Amount of adsorbed molecules or an adsorption layer on the solid surfaces is determined by the vapour pressure in the ambient air.

Let us consider a solid plane in a contact with a vapour in the ambient air. As we already mentioned the liquid vapour adsorbs on the solid surface. In the case of low adsorption (no polymolecular adsorption) the dependency of the adsorption on the vapour pressure in the ambient air, $p$, is described by the Langmuir isotherm. The latter is written below in the following form:

$$
\Theta=\frac{\left(p / p^{*}\right) \exp \left(-\frac{\Delta U}{k T}\right)}{1+\left(p / p^{*}\right) \exp \left(-\frac{\Delta U}{k T}\right)},
$$

where $\Theta$ is the fraction of solid substrate covered by adsorbed vapour molecules; $k$ and $T$ are the Boltzmann constant and absolute temperature in Kelvin, respectively; $p^{*}$ is a characteristic vapour pressure and $\Delta U$ is the difference between the free energy of vapour molecules in adsorbed state and in the vapour (see [18] for example). If $\Delta U / k T>>1$, that is the energy of adsorbed molecules is much higher than the energy of free vapour molecules (or water molecules are infinitely repelled 
from the solid surface). Hence, the interaction between water molecules in the droplet is infinitely stronger than the repulsion between water molecules and solid substrate. Only in this case $\Theta \approx 0$ no adsorption takes place. In this and only this particular case the water droplet forms $180^{\circ}$ contact angle with the solid substrate (see Fig. 3a). We refer to this case as the complete non-wetting case.

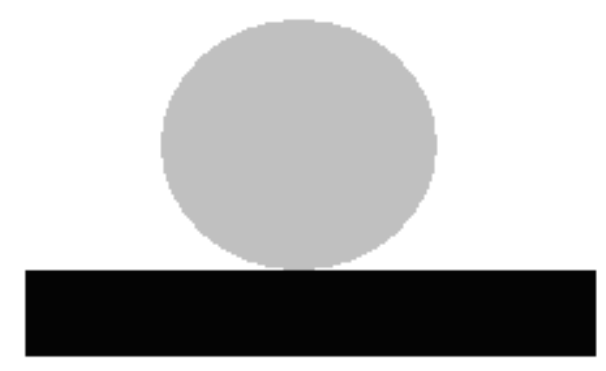

Fig. 3a. Water droplet on ideal complete non-wetting solid substrate, contact angle is equal to $180^{\circ}$ : interaction between water molecules is much stronger than interaction between water molecules and the solid substrate. No adsorption of water molecules on the solid substrate.

Surprisingly there is no surfaces in the nature, which are completely non-wet by water. Even on very hydrophobic solid, Teflon, water droplets form the contact angle in the range of $120^{\circ}$. The latter means that the condition $\Delta U / k T \gg 1$ is not satisfied and adsorption of water molecules even on Teflon substrate is unavoidable.

The latter means that we came to a very interesting conclusion: there is a strong link between adsorption of water molecules on solid substrates and the contact angles. Hence, the real picture should include adsorption of water vapour on non-wettable solids (Fig. 3b)! 


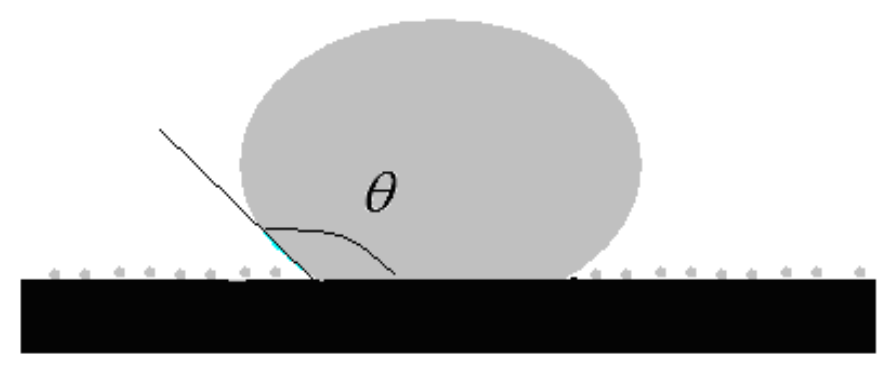

Fig. 3b. Water droplet on a real non-wettable substrate. Contact angle, $\theta$, is less than $180^{\circ}$, and, hence, adsorption of water molecules on the solid substrate is unavoidable!

Now let us look on the adsorption of water molecules from a different point of view. Let the original interfacial tension of bare solid substrate be $\gamma_{s v}^{0}$. After adsorption of water molecules the new interfacial tension becomes $\gamma_{s v}^{a}$, which can be either higher or lower than the original interfacial tension $\gamma_{s v}^{0}$. After the fraction $\Theta$ of the solid substrate is covered by adsorbed molecules the new interfacial tension, $\gamma_{s v}$, can be presented in the following form:

$\gamma_{s v}=\gamma_{s v}^{0}(1-\Theta)+\gamma_{s v}^{a} \Theta$

Comparison of the latter equation and Langmuir Eq. (1) shows that the solid liquid interfacial tension, $\gamma_{s v}$, is not a unique property of the solid substrate but depends on the vapour pressure in the ambient air, $p$, that is, $\gamma_{s v}(p)$. Is this dependency a strong one? There is no direct way to measure solid-vapour interfacial tension and its dependency on the vapour pressure in the case of hydrophobic or even hydrophilic substrates. However, in the case of partial and complete wetting (see below) there is a completely different way of thinking, which allows calculating that new interfacial tension, $\gamma_{s v}(p)$.

In the case of non-wetting substrates adsorption of water molecules is small because the interaction between water molecules is substantially stronger than the 
interaction between water molecules and the solid substrate. Such surfaces are referred to as "low energy surfaces". In this case adsorption of water molecules on the solid substrates is small because adsorption results in a higher interfacial tension $\gamma_{s v}$ as compared with the initial interfacial tension, $\gamma_{s v}^{0}$. However, according to the Boltzmann distribution still the adsorption is unavoidable even in this extreme case and latter manifests itself in a contact angle lower than $180^{\circ}$.

In the case of partial or complete wetting the situation is very much different. In this case adsorption of water molecules on the solid substrate results in a lowering of the solid-vapour interfacial tension and even more than that, the adsorption results in a polymolecular adsorption, that is in a formation of a thin adsorbed water film on the solid substrate. There is no need to say that the interfacial tension can not be refereed to any more as a solid-vapour interfacial tension but rather as a solid-liquid film-vapour interfacial tension.

We have to remind that there is no experimental way to determine independently the solid-vapour interfacial tension and the latter becomes even more sophisticated in the case of the presence of adsorbed films on the solid substrate. Fortunately, there is a completely different way of thinking, which allows us to do so!

\section{Equilibrium contact angle in the case of partial wetting}

What is required for a complete description of a fluid shape at the equilibrium with a solid when we reach the range of $100 \mathrm{~nm}$ thickness inside droplet (Fig. 4)?

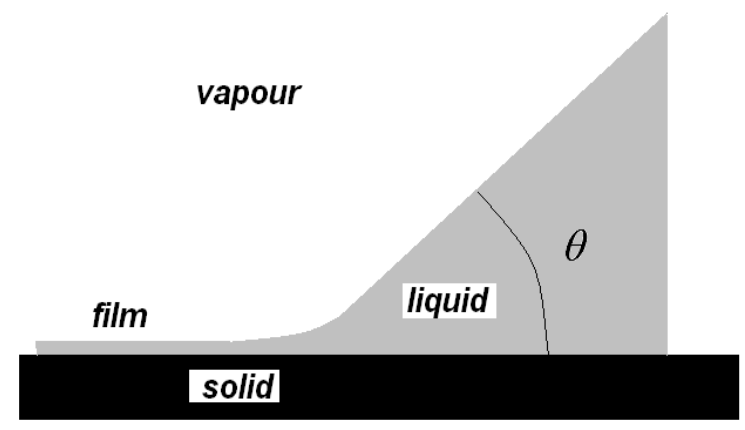

Fig. 4. Liquid in a contact with solid, vapour and liquid films in front. $\theta$ is the macroscopic contact angle (measured inside the liquid). 
At the equilibrium the chemical potentials of liquid molecules in liquid droplet/meniscus, vapour and adsorbed liquid film on the solid surface should be equal. The latter means that the following three equilibrium conditions should be satisfied:

(a) the liquid in the droplet must be at equilibrium with its own vapour;

(b) the liquid in the droplet must be at equilibrium with the solid;

(c) the vapour must be at equilibrium with the solid substrate. The latter, as we already showed above, results in the formation of adsorption films on the solid surface.

All three mentioned above equilibrium conditions mean that chemical potential of liquid molecules in the liquid, vapour and adsorbed film should be equal.

Unfortunately, frequently either all three equilibrium conditions (a), (b), (c) or part of them have been ignored in a number of publications and still the conditions have been referred to as "equilibrium". The first requirement (a) results in the equality of chemical potentials of the liquid molecules in the vapour and inside the droplet. The latter results in the well-known Kelvin equation for the excess pressure, $P_{e}$ :

$$
P_{e}=\frac{R T}{v_{m}} \ln \frac{p_{s}}{p}
$$

where $v_{m}$ is the molar volume of the liquid, $p_{s}$ is the pressure of the saturated vapour at the temperature $T, R$ is the gas constant, $p$ is the vapour pressure which is at the equilibrium with the liquid droplet, the excess pressure $P_{e}=P_{a}-P_{l}$, where $P_{l}$ is the pressure inside the liquid and $P_{a}$ is the pressure in the ambient air. The latter equation determines the unique equilibrium excess pressure $P_{e}$ and, hence, the unique radius of curvature of the equilibrium droplet:

$$
\mathfrak{R}=-\frac{2 \gamma}{P_{e}}
$$

We remind now that the excess pressure inside the drop, $P_{e}$, should be negative (pressure inside the droplet is bigger than the pressure in the ambient air). That means that the right hand side in Eq. (3) should be negative also. The latter is negative only if $p>p_{s}$ (according to Eq. 3), that is, the droplets can be at the equilibrium only with the oversaturated vapour! Note, the equilibration process goes for sufficiently long time (hours) and it is necessary to keep oversaturated vapour 
over a solid substrate under investigation until the equilibrium is reached. There are only a limited number of attempts to measure drops under condition of oversaturation [19]. The latter means that it is difficult to investigate experimentally equilibrium droplets on the solid substrate. However, there is a flood of investigations published in the literature on measurements of "equilibrium contact angles" of droplets on solid substrate. The previous consideration shows that contact angles measured are not at equilibrium at all: actually in most investigations a static advancing contact angle was measured, which can be very far from the equilibrium one (see below). Note, evaporation takes place most vigorously in a vicinity of the three phase contact line (see below), which makes a relation of the measured contact angle with the equilibrium one even more questionable.

Now we consider requirements of equilibrium (b) and (c). Let us assume that we can create at least theoretically an oversaturated vapour over the solid substrate and wait long enough until the equilibrium is reached. Now the liquid molecules in the vapour are at the equilibrium with the liquid molecules in the droplet. The presence of adsorbed liquid layers on the solid substrate results in the case of partial wetting in lower surface tension as compared with the surface tension of the bare solid surface, $\gamma_{s v}^{0}$, because the presence of liquid molecules on the surface changes the initial surface tension. The presence of adsorbed liquid films results in the formation of a new interfacial tension, $\gamma_{\text {suh }_{e}}$, where $h_{e}$ is the thickness of the adsorbed layer.

In Fig. 5 an equilibrium liquid droplet is presented in the contact with equilibrium adsorbed liquid film on the solid surface. What happens in a vicinity of the line where they meet? 


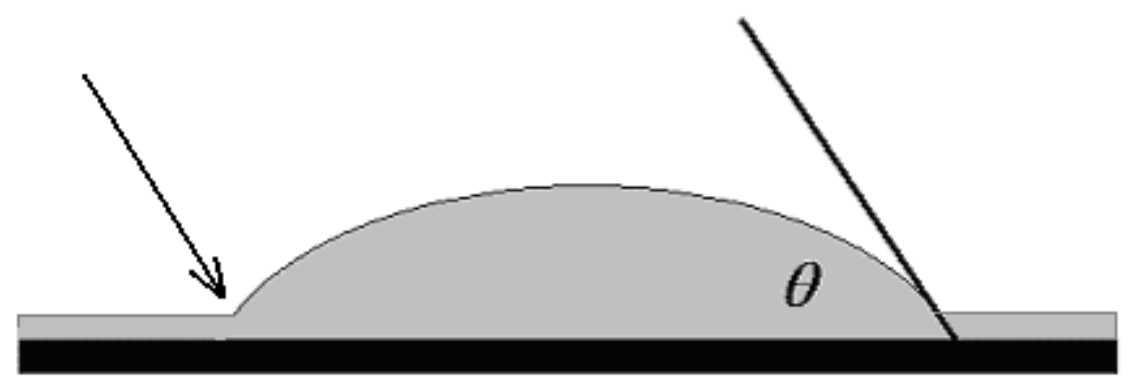

Fig. 5. Cross section of an equilibrium liquid droplet (at oversaturation) in the contact with an equilibrium adsorbed liquid film on the solid substrate. What happens on the line (shown by an arrow) where they meet?

Is the situation presented in Fig. 5 possible? The answer is 'no' because such sharp transition from the liquid droplet to the liquid film is impossible: on the line shown by the arrow the capillary pressure will be infinite. Hence, it should be a smooth transition from the flat equilibrium liquid film on the solid surfaces to the spherical droplet, as shown in Fig. 6, where such smooth transition is shown.

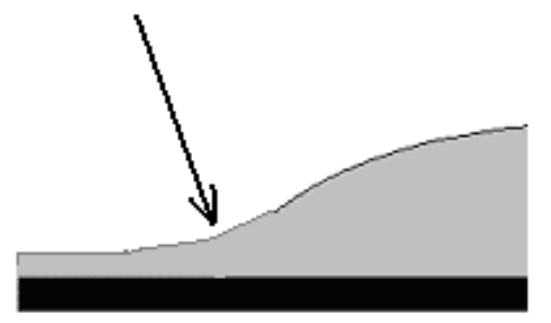

Fig. 6. Transition zone from the flat equilibrium liquid film on a solid surface to the liquid droplet. The arrow shows the point where to the left the liquid profile is concave and to the right the profile is convex. 


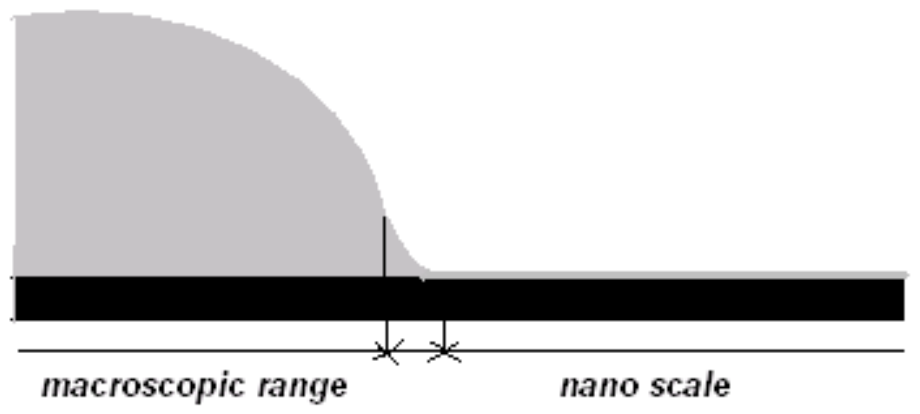

Fig. 7. Transition from macroscopic droplet to thin film in front via intermediate (transition) region.

Let us call this region, where transition from a flat film to the droplet takes place, a transition zone (Fig. 7). The presence of the transition zone shows that pure capillary forces are unable to keep the liquid in this zone at the equilibrium: the liquid profile is convex (hence, the capillary pressure under the liquid surface is higher than in the ambient air) to the right from the arrow in Fig. 6, and the liquid profile is concave (hence, the capillary pressure under the liquid surface is lower than in the ambient air) to the left from the arrow in Fig. 6. The shape of the profile inside the transition zone is determined by the surface forces action [9].

The most important conclusion is as follows [9]: the nature of surface forces acting in thin layers and the transition zones (Figs. 5, 6, 7) determines the equilibrium of liquid droplets on solid surfaces.

Before considering the surface forces action in a vicinity of the three phase contact line let us consider what should happen in the case of non-volatile liquid, when the formation of adsorbed layers is slow. Usually, low volatility means liquids with big molecules, the latter means high viscosity and correspondingly higher time scale of equilibration process with the oversaturated vapour. In spite of that let us assume that the liquid is non-volatile. In the case of partial wetting, as we already saw, at equilibrium liquid droplets can not be at equilibrium with a bare solid surface but at the equilibrium with an adsorption layer of the liquid molecules on the solid substrate in front of the droplet. If the liquid is volatile then the latter layer is created by means of evaporation-adsorption. However, if the liquid is non-volatile or low volatile then the same layer will be created by means of flow from the droplet edge 
onto the solid substrate. As a result the solid substrate will be eventually covered at equilibrium by equilibrium liquid film of thickness $h_{e}$. The thickness of the equilibrium liquid film, $h_{e}$, is determined (as we see below) by the potential of surface forces action. Characteristic time scale of this process is hours, because it is determined by the flow in the thinnest part in the vicinity of the apparent three phase contact line, where the viscous resistance is very high. During those hours evaporation of the liquid from the droplet can not be ignored and we go back to the problem of volatility.

Let us assume, however, that the equilibrium film after all forms in front of the liquid droplet and we waited enough for the equilibrium. Now the have again three following interfacial tensions: $\gamma, \gamma_{s l}$, and $\gamma_{s v h_{e}}$, which are interfacial tensions of the following interfaces liquid -vapour, solid - liquid and solid substrate covered with the liquid film of thickness $h_{e}$ - vapour. We came back to the same interfacial tensions as in the case of volatile liquid. We can not measure the interfacial tension $\gamma_{s v h_{e}}$ directly. However, there is a solution to this problem, which is presented below.

Thin liquid films on the solid substrate and solid-liquid interfacial tension

The excess free energy, $\Phi_{f}$, per unit area of a flat equilibrium liquid film of thickness $h_{e}$ on a solid substrate at the equilibrium with the vapour in the surrounding air is equal to

$\Phi_{f}=\gamma+P_{e} h_{e}+f_{D}\left(h_{e}\right)+\gamma_{s l}-\gamma_{s v}^{0}$

where $f_{D}\left(h_{e}\right)$ is the excess free energy due to the action of surface forces.

Because of the equilibrium of the liquid film with the vapour the excess pressure, $P_{e}$, can not be left as an arbitrary constant: it is determined by the equality of chemical potentials of liquid molecules in the film and in the vapour. The latter requirement results in the well-known Kelvin's equation (3).

The excess free energy (5) is a function of $h_{e}$, which is the thickness of the equilibrium film. The volume of the liquid film is $V=h_{e} S$, where $S$ is the total area covered by the film. Hence, the usual conditions of the thermodynamic equilibrium should hold, which give a minimum to the excess free energy (5). Those conditions 
are as follows: $\frac{d \Phi_{f}}{d V}=0, \frac{d^{2} \Phi_{f}}{d V^{2}}>0$. Taking into account that $S$ is constant the latter condition can be rewritten as $\frac{d \Phi_{f}}{d h_{e}}=0, \frac{d^{2} \Phi_{f}}{d h_{e}^{2}}>0$. The first requirement results in $P_{e}=\Pi\left(h_{e}\right)$,

and the second requirement yields

$\frac{d \Pi\left(h_{e}\right)}{d h_{e}}<0$

where $\Pi(h)=-\frac{d f_{D}(h)}{d h}$ is referred to as Derjaguin's pressure. Derjaguin's pressure, $\Pi(h)$, is the physical property, which can be experimentally measured (see for example [4-7]). Using the latter definition we can rewrite the excess free energy $f_{D}(h)$ as: $f_{D}(h)=\int_{h}^{\infty} \Pi(h) d h$.

As we already noticed in the Introduction, the excess free energy of thin liquid films, $f_{D}(h)$, includes contributions of the same components of surface forces as in the case of interaction of colloidal particles: van der Waals, electrostatic, steric, interaction caused by the orientation of water dipoles at water-solid and water-vapour interfaces. However, there is one very important difference: interaction between identical colloidal particles or droplets is symmetrical, while the colloidal forces in thin liquid films are highly asymmetrical because two completely different interfaces are involved: liquid -solid and liquid-vapour.

Eq. (6) determines the thickness of the equilibrium liquid film, $h_{e}$, via Derjaguin's pressure isotherm. Eq. (7) gives the well-known stability condition of flat equilibrium liquid films $[4,9]$.

According to the modern theory of surface forces the following types of Derjaguin's pressure are known and presented in Fig. 8. 


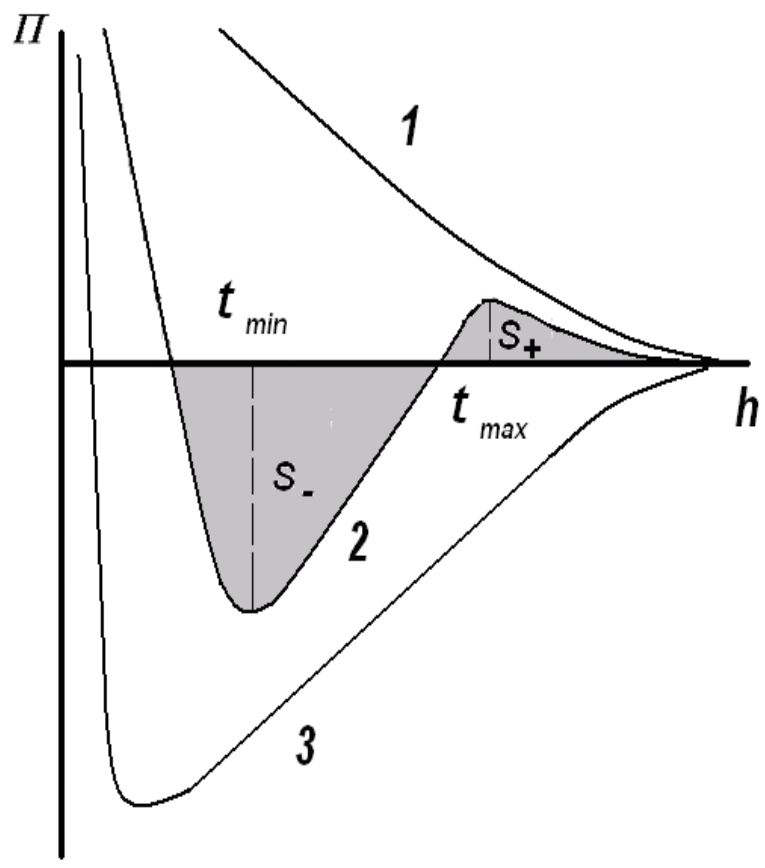

Fig. 8. Types of isotherms of Derjaguin's pressure: 1 - complete wetting, observed for oil films on quartz, glass, metal surfaces; 2 - partial wetting, observed for aqueous films on quartz, glass, metal surfaces; 3 - non-wetting case, which actually has never been measured.

According to the stability condition (7) all flat equilibrium films are stable in the case of complete wetting (curve 1, Fig. 8) and only films are stable in the range of thickness from 0 to $t_{\min }$ (these films are referred below as $\alpha$-films, which are absolutely stable, see below) and at $h>t_{\max }$ (the latter films are referred below as $\beta$ films and it is shown below that they are meta-stable) in the case of partial wetting (curve 2 in Fig. 8). Hence, only those - $\alpha$ - and $\beta$ - films can exist as flat films.

Note, s-shaped Derjaguin's pressure isotherms (curve 2 in Fig. 8) are characteristic shapes of thin films of water and aqueous solutions. All properties of water and aqueous solutions are vitally important for our life. The latter means that the peculiar shape of Derjaguin's pressure of water and aqueous solutions, presented in Fig. 8 curve 2 in some unknown way determines the existence of life. At the moment we do not know in which way it does but the peculiar shape of curve 2 in Fig. 8 tell us something what we are currently unable to decode. 
Now we can rewrite the expression for the excess free energy of the film (5) using the Derjaguin's pressure in the following way:

$\Phi_{f}=\gamma+P_{e} h_{e}+\int_{h_{e}}^{\infty} \Pi(h) d h+\gamma_{s l}-\gamma_{s v}^{0}$.

The latter expression gives the excess free energy via a measurable physical dependency, $\Pi(h)$, which is the Derjaguin's pressure isotherm.

We can rewrite the latter expression (8) of the excess free energy of thin liquid films as

$\Phi_{f}=\gamma_{s v h_{e}}-\gamma_{s v}^{0}$

where

$\gamma_{s v h_{e}}=\gamma+P_{e} h_{e}+\int_{h_{e}}^{\infty} \Pi(h) d h+\gamma_{s l}$

is the "interfacial tension" (actually the excess free energy) of the solid substrate covered with the liquid film of thickness $h_{e}$. Note, the latter expression for the interfacial tension of solid covered by the equilibrium liquid film-vapour has nothing to do with bare solid-vapour interfacial tension.

The latter expression determines the unknown value of $\gamma_{s v h_{e}}$ into the Young's equation:

$\cos \theta_{e}=\frac{\gamma_{s v h_{e}}-\gamma_{s l}}{\gamma}$

Combination of Eqs. (10) and Eq. (11) results in

$\cos \theta_{e}=\frac{\gamma+P_{e} h_{e}+\int_{h_{e}}^{\infty} \Pi(h) d h}{\gamma} \approx 1+\frac{1}{\gamma} \int_{h_{e}}^{\infty} \Pi(h) d h$,

because the term $\frac{P_{e} h_{e}}{\gamma}$ is usually small as compared with other terms in Eq. (12) [9]. 
The latter equation is a well-known Derjaguin-Frumkin equation for the equilibrium contact angle, which has been deduced using a different thermodynamic consideration [4]. The later was deduced from the more rigorous consideration of equilibrium conditions [9].

Note, there are numerous publications where Young's equation, which was deduced for equilibrium was applied for highly non-equilibrium situation: instead of the "interfacial tension" (the excess free energy) of the solid substrate covered with the liquid film of thickness $h_{e}, \gamma_{s v h_{e}}$ the authors used $\gamma_{s v}^{0}$, that is, the interfacial tension of the bare solid substrate, which is not covered by adsorption layer. The latter is in a contradiction with the thermodynamics: the requirements of equilibrium between the vapour and solid substrate and the liquid and solid substrate in front are both violated.

In the case of partial wetting (water and aqueous solutions) $-1<\cos \theta_{e}<1$. From that condition we conclude that the integral in the right hand side of Eq. (12) should be negative. The latter requirement is satisfied in the case of partial wetting (see curve 2 in Fig. 8) if

$\int_{h_{e}}^{\infty} \Pi(h) d h<0$

The latter inequality is satisfied if

$S_{-}>S_{+}$,

see Fig. 8 curve 2.

\section{Hysteresis of contact angle on smooth homogeneous substrates}

The previous consideration shows that all droplets deposited on a solid substrate are in a non-equilibrium state at oversaturation. According to usually adopted deposition procedure the resulting contact angle is a static advancing contact angle. That is, consideration of static advancing contact angle and it connection with surface forces action is the important problem in this area.

The above derivation of Eq. (12) shows, that the latter equation determines only one unique equilibrium contact angle. Static hysteresis of contact angle results in an infinite number of "quasi-equilibrium contact angles" of the drop on the solid surface, 
not the unique contact angle, $\theta_{e}$, but the whole range of contact angles, $\theta_{r}<\theta_{e}<\theta_{a}$, where $\theta_{r}$ and $\theta_{a}$ are static receding and advancing contact angles, correspondingly.

Let us explain the meaning of static advancing and receding contact angles. For that purpose let us consider a liquid droplet on a horizontal substrate, which is slowly pumped through an orifice in the solid substrate (Fig. 9). Using the latter experimental arrangement static advancing and receding contact angles can be investigated [20].

Let us assume that in some way an initial contact angle of the droplet was equal to the equilibrium one.

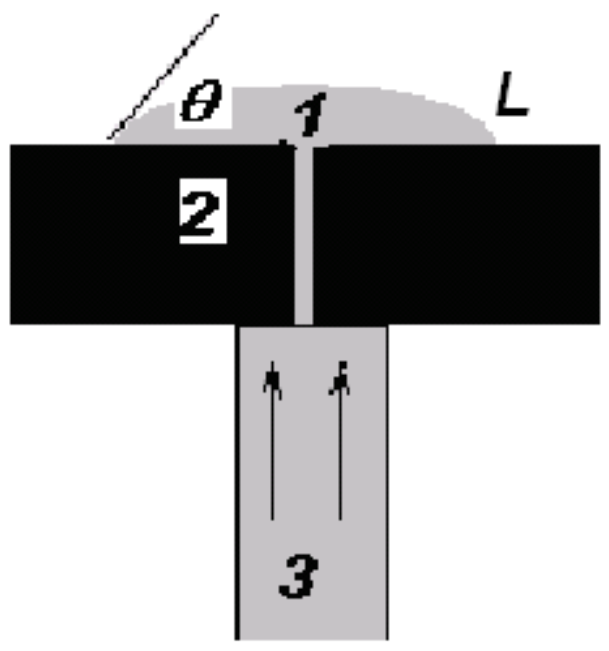

Fig. 9. Schematic presentation of a liquid droplet on a horizontal solid substrate, which is slowly pumped through the liquid source in the drop centre. $L$ - radius of the drop base; $\theta$ - contact angle; 1 - liquid drop, 2 - solid substrate with a small orifice in the centre, 3 - liquid source (syringe).

Let us start carefully and slowly pumping the liquid through an orifice in the centre. Contact angle will grow, however, the radius of the drop base will not change until a critical value on the contact angle, $\theta_{a}$, is reached. Further pumping will result in a drop spreading.

If we start from the same equilibrium contact angle and start to pump out the liquid through the same orifice, then again the contact angle will decrease but the droplet will not shrink until the critical contact angle, $\theta_{r}$, is reached. After that the 
droplet will start to recede. Note, in this case it is possible to start from an advancing contact angle and the identical receding contact angle will be obtained in the end.

For example, in the case of water droplets on a smooth homogeneous specially treated for purity glass surface: $\theta_{r} \sim 0^{\circ}-5^{\circ}$, while $\theta_{a}$ is in the range of $40^{\circ}$ $60^{\circ}$.

It is usually believed that the static hysteresis of contact angle is determined by the surface roughness and/or heterogeneity. No double that a roughness and/or a chemical heterogeneity of the solid substrate contribute substantially to the contact angle hysteresis. However, there are numerous experimental evidences in the literature that the static hysteresis of contact angle exist even on smooth homogeneous solid substrates. Even more than that, the static hysteresis of contact angle is present even on surfaces which are definitely molecularly smooth: free liquid films [21, 22]. Note, the existence of a hysteresis of contact angle on a smooth homogenous substrate is in a contradiction with the prediction based on Young's equation, $\cos \theta=\frac{\gamma_{s v}-\gamma_{s l}}{\gamma}$, because according to that equation there is not any hysteresis on smooth homogeneous surfaces.

Let us remind that in a vicinity of the apparent three phase contact line surface forces (Derjaguin's pressure) disturb substantially the liquid profile and the latter results in a flow of the liquid from the droplet onto the substrate in a vicinity of the apparent three phase contact line. The latter means that the liquid edge is always in a contact with already wetted rough solid substrate. Equilibrium and hysteresis contact angles on rough surfaces has never been considered from this point of view before and are the subject of future investigations.

We suggested earlier [8,9] a completely new concept of hysteresis of contact angle on smooth homogeneous substrates. Below we give a qualitative description of the phenomenon.

On smooth homogeneous solid substrate any contact angle, $\theta$, in the range $\theta_{r}<\theta<\theta_{a}$ is different from the equilibrium one. Hence, the liquid droplet cannot be at the equilibrium but in the state of a very slow "microscopic" motion. More detailed observations and theoretical considerations shows $[8,9]$ that any contact angle different from the equilibrium one, $\theta_{e}$, the liquid droplet is in a state of slow "microscopic motion", which is located in a tiny vicinity of the apparent three phase 
contact line. The latter motion abruptly becomes "macroscopic motion" after a critical contact angles $\theta_{a}$ or $\theta_{r}$ are reached.

The presence of the contact angle hysteresis shows that the actual equilibrium contact angle is very difficult to obtain experimentally even if we neglect the equilibrium with vapour and solid substrate.

On this stage we are capable of explaining the nature of the hysteresis of contact angles via s-shape of isotherm of Derjaguin's pressure in the case of partial wetting (curve 2 in Fig. 8). Consideration of the advancing contact angle in the case of droplet is given in [8], below we consider the static hysteresis of contact angle in the case of a capillary to simplify our consideration.

Let us consider a capillary meniscus in the case of partial wetting in a capillary. Keep in mind that the capillary is in a contact with a reservoir, where the pressure, $P_{a}-P_{e}$, is kept, that is the pressure in the reservoir is lower than the atmospheric pressure, $P_{a}$.
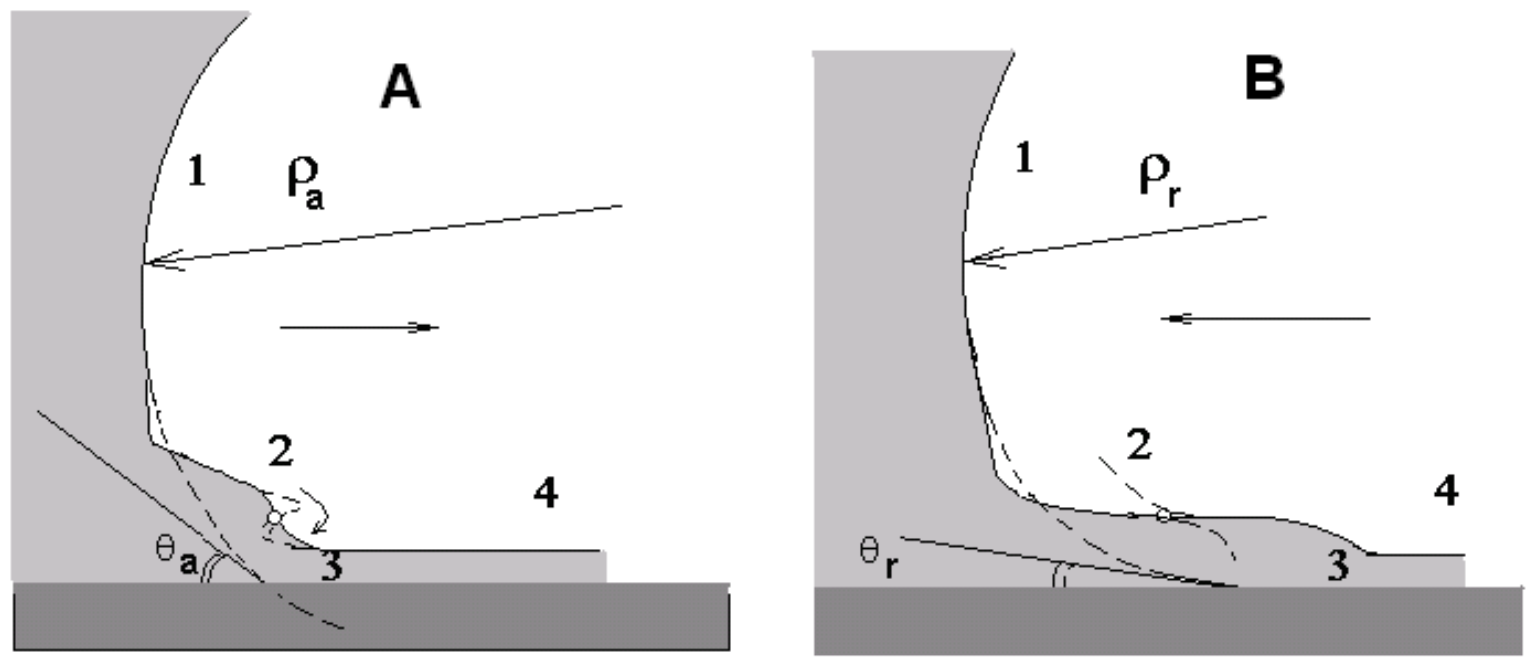

Fig. 10. Hysteresis of contact angle in capillaries in the case of partial wetting (sshaped isotherm of Derjaguin's pressure). A - advancing contact angle. 1- a spherical meniscus of radius $\rho_{a}, 2$ - transition zone with a "dangerous" marked point (see explanation in the text) , 3 - zone of flow, 4 - flat films. Close to the marked point a dashed line shows the profile of the transition zone just after the contact angle reaches the critical value $\theta_{a}$, a beginning of a "caterpillar motion". B - receding contact angle. 1- a spherical meniscus of radius $\rho_{r}<\rho_{a}, 2$ - transition zone with a 
"dangerous" marked point (see explanation in the text), 3 - zone of flow, 4 - flat films. Close to the marked point a dashed lines show the profile of the transition zone just after the contact angle reaches the critical value $\theta_{a}$.

If we increase the pressure under the meniscus then the meniscus does not move but changes its curvature to compensate the excess pressure and, as a consequence, the contact angle increases accordingly. The meniscus does not move until some critical pressure and critical contact angle, $\theta_{a}$, are reached. After further increase in pressure the meniscus starts to advance. A similar phenomenon takes place if we decrease the pressure under the meniscus: it does not recede until a critical pressure and corresponding critical contact angle, $\theta_{r}$, are reached. The latter means that in the whole range of contact angles, $\theta_{r}<\theta<\theta_{a}$, the meniscus does not move macroscopically. It is obvious that on the smooth homogeneous solid substrate only one contact angle corresponds to the equilibrium position and all the rest do not.

Explanation of the hysteresis of contact angle on smooth homogeneous solid substrates is based on s-shaped isotherm of Derjaguin's pressure in the case of partial wetting. This shape determines a very special shape of the transition zone in the case of equilibrium meniscus $[8,9]$. In the case of increasing of the pressure behind the meniscus (Fig. 10A) a detailed consideration [8,9] of the transition zone shows: close to the "dangerous" point marked in Fig. 14A, the slope of the profile becomes steeper with increasing pressure. In the range of very thin films (region 3 in Fig. 10A) there is a zone of flow. Viscose resistance in this region is very high, that is why the advancing of the meniscus proceeds very slowly. After some critical pressure behind the meniscus is reached then the slope at the "dangerous" point reaches $\pi / 2$, after that the flow step-wisely occupies the region of thick films the fast "caterpillar" motion starts as shown in Fig.10A.

In the case of decreasing the pressure behind the meniscus the event proceed according to Fig. 10B. In this case again up to some critical pressure the slope in the transition zone close to the "dangerous" marked point becomes more and more flat. In the range of very thin films (region 3 in Fig. 10B) there is a zone of flow. Viscose 
resistance in this region again is very high, that is why the receding of the meniscus proceeds very slowly. After some critical pressure behind the meniscus is reached then the profile in the vicinity of the "dangerous" point shows the discontinuous behaviour, which is obviously impossible. That means the meniscus will start to slide along thick $\beta$-film. That is, the meniscus will move relatively fast leaving behind the thick $\beta$-film. The latter phenomenon (the presence of a thick $\beta$-film behind the receding meniscus of aqueous solutions in quartz capillaries) has been discovered experimentally [23-25]. The latter phenomenon (illustrated by the presence of a thick $\beta$-film behind the receding meniscus in aqueous solutions in quartz capillaries) has been discovered experimentally and supports our arguments explaining static contact angle hysteresis on smooth homogeneous substrates.

In the process of deposition of droplets the latter reach the final position after the contact angle reaches the value, which corresponds to the static advancing contact angle. The latter was considered in [8] based on the consideration of events in a vicinity of the apparent three phase contact line. The latter consideration revealed that the static advancing contact angle in the case of droplets substantially differs from the corresponding static advancing contact angle in thin capillaries: in the case droplets the latter is not a unique property of droplet-solid substrate system but depends on the droplet volume. It was shown that the advancing contact of droplet increases with a decrease of the droplet volume. The latter theoretical conclusion was directly experimentally confirmed in [26-27].

Consideration in this section shows that the events in a vicinity of the three phase contact line are rather sophisticated. The latter explain why there is no reasonable theory of kinetics of spreading/dewetting in the case partial wetting based on the consideration of surface forces (Deriaquin's pressure) in a vicinity of the three phase contact line. The latter is a very important problem for the future research.

\section{Kinetics of spreading and surface forces}

The consideration above shows that there is not enough understanding of importance of surface forces action even in the case equilibrium/quasi-equilibrium in the area of wetting phenomena. It is a reason why a number of approaches have been developed, which attempt to describe kinetics of wetting without consideration 
of surface forces acting in a vicinity of the moving three phase contact line. Those approaches have been reviewed recently in [28]. Note the common feature of all those approaches discussed in [28] is ignoring the surface forces action in a vicinity of the moving three phase contact line.

The frequently used approach is that based on T.D. Blake, J.M. Haynes original model [29] and its further modifications [30-32]. The latter model was applied for the case electrowetting [32], which itself is an interesting area, however, not reviewed below.

The surface diffusion, which according to [29] is the driving force of spreading, is no doubt an important phenomenon, which should be included into consideration. To the best of the author's knowledge the only attempt in this direction was undertaken in [33] for a special case. Extension of the approach suggested in [33] for the more general case of both complete and partial wetting is an important problem for the future. The idea suggested in [33] is as follows: the surface diffusion results in an effective slippage, which removes a friction singularity on the moving three phase contact line.

The previous consideration in the case of equilibrium and quasi-equilibrium allows application of the same approach based on the consideration of surface forces action in a vicinity of the moving three phase contact line to the case of kinetics of wetting.

Let us consider in more details the vicinity of the moving contact line, which is magnified in Fig. 11.

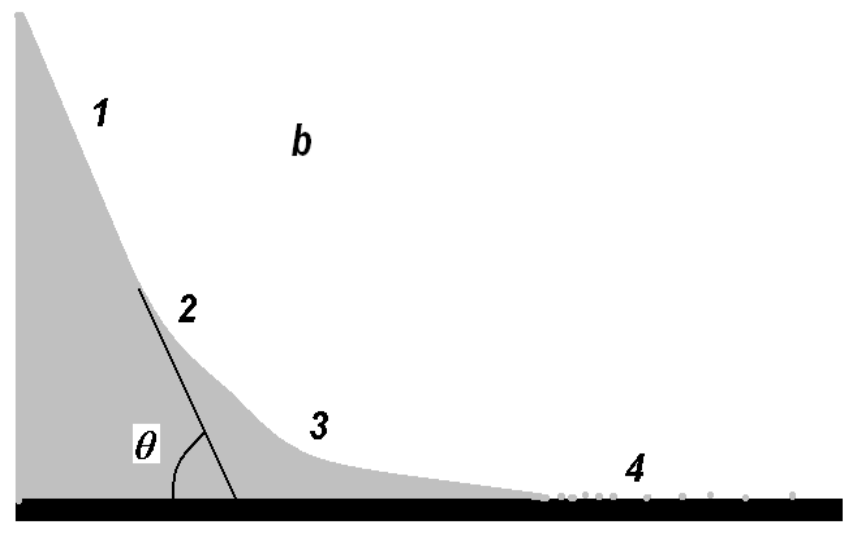


Fig. 11. A magnification of the vicinity of the moving apparent three phase contact line in the case of complete wetting: 1 - spherical part of the drop, which forms a dynamic contact angle, $\theta$, with the solid substrate; 2 - a region, where a spherical shape is distorted by the hydrodynamic force; 3 - a region, where Derjaguin's pressure comes into play and become increasingly important towards the end of the region 3; 4 - a region, where a macroscopic description is not valid any more and surface diffusion takes place.

The whole vicinity of the three phase contact line can be subdivided into four regions (Fig. 11) [9]. The region 1 is a spherical meniscus in the main part of the spreading droplet. This region is included to show the dynamic contact angle, $\theta(t)$, which is defined at the intersection of the tangent to the spherical part of the droplet with the solid substrate. The dynamic contact angle is unknown and should be determined by matching of all regions presented in Fig. 11. Inside the next region, 2, the spherical shape is distorted by the hydrodynamic flow. This region is followed by region 3, where Derjaguin's pressure comes into play. Over the region 3 Derjaguin's pressure action becoming increasingly important as compared with the capillary forces. Towards the end of the region 3 the Derjaguin's pressure overcomes the capillary forces and becomes the only driving force of the spreading process. The region 3 is followed by region 4 . In this region a macroscopic description of the spreading process becomes impossible because the characteristic scale in the vertical direction is of the order of the molecular size. We refer to region 4 as the region of surface diffusion.

The spreading of an axi-symmetric liquid drop on a solid plane solid substrate in the case of complete wetting was considered theoretically and compared with available experimental data [34]. Both capillary and Derjaguin's pressure were taken into account [34]. 


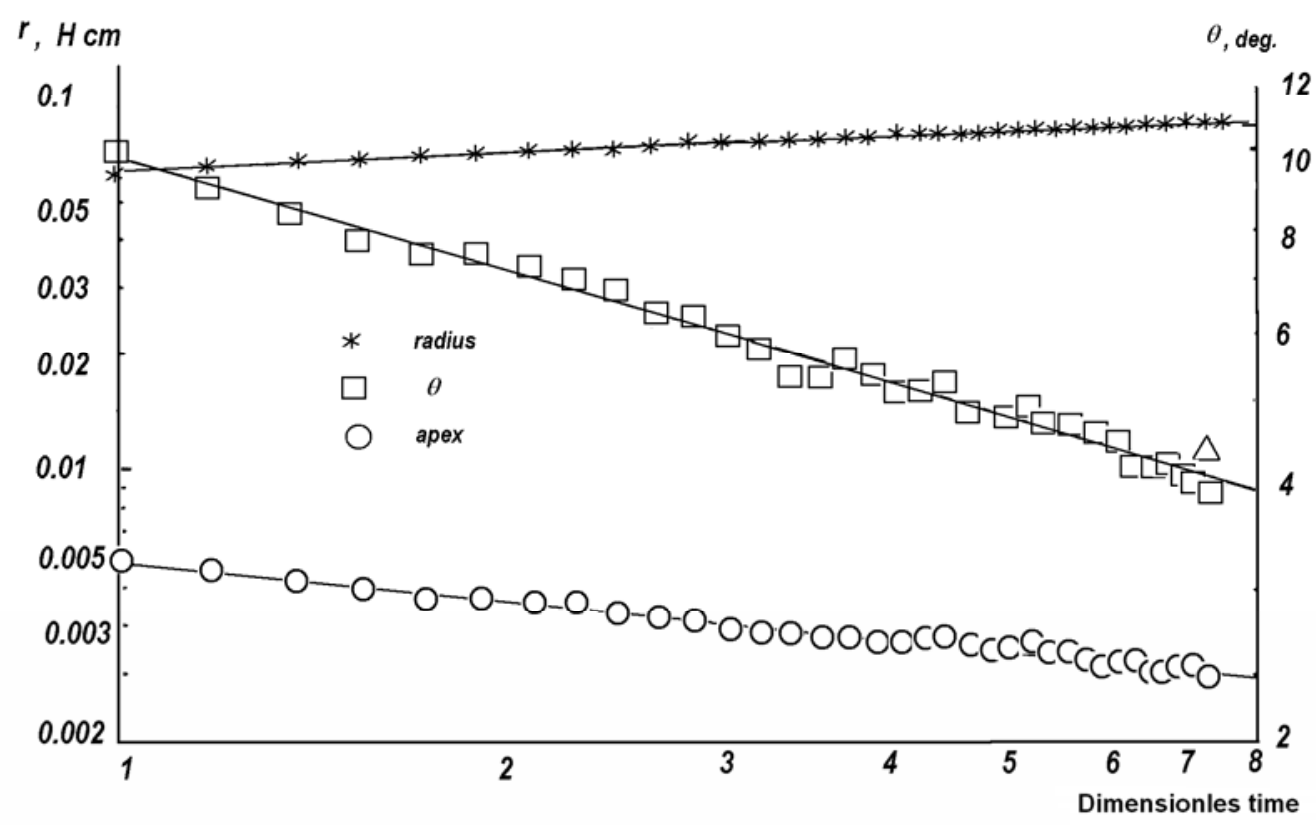

Fig. 12. Experimental dependencies of radius of spreading, dynamic contact angle and the drop apex height on time in the case complete spreading. Solid lines are theoretical dependences [34]. The comparison shows an excellent agreement between the theory predictions and experimental data.

However, in spite of a very good agreement between the experimental data and the theoretical treatment there are two important mathematical problems left even in the case of complete wetting. The fist problem is a matching of outer (spherical droplet) and inner (thin liquid films) asymptotic solutions. In [33] a patching of asymptotic solutions was used. The second problem is the divergence of the total liquid volume in the outer solution: that volume was supposed to be a very small. The latter problem is related to the infinite region of integration of the outer solution and will completely disappear if the surface diffusion is taken into account properly: in this case the region of integration will be finite.

The major problem left in this area is a development of the approach, which takes into account the surface forces action in the case of partial wetting.

4. Spreading over porous substrates 
Kinetics of spreading and simultaneous imbibitions of the liquid into porous media is a process of a substantial industrial importance. Surprisingly the latter process drew the attention of a scientific community relatively recently (see recent reviews on the subject [35-36]). A schematics of the process presented in Fig. 13 in the case of spreading over thin porous layer.

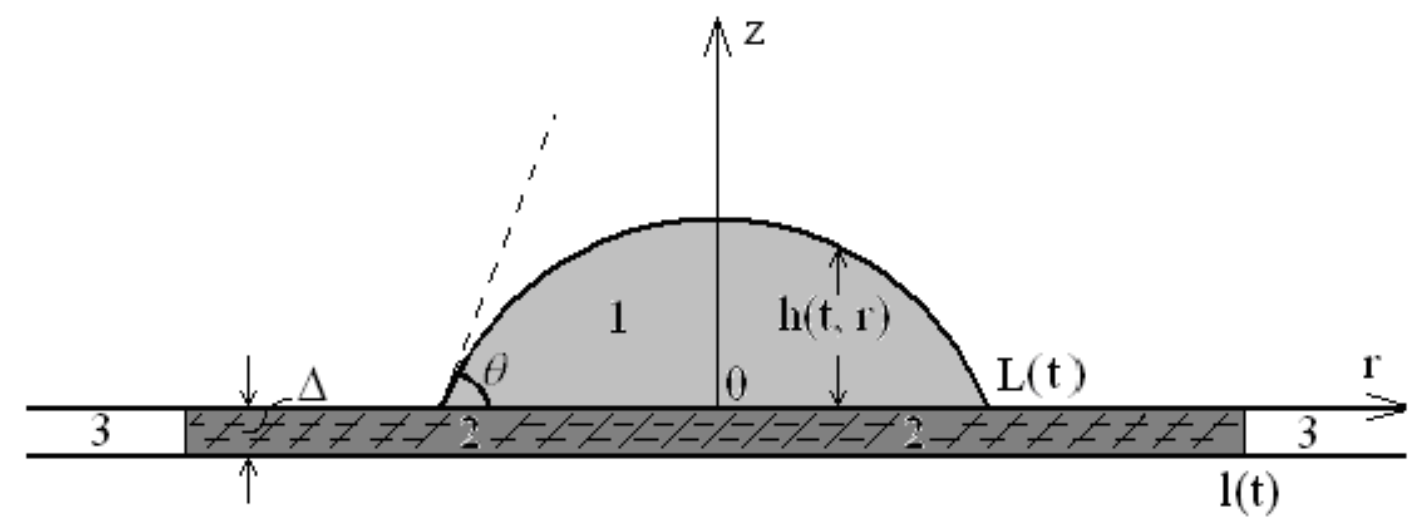

Fig. 13. Cross-section of an axi-symmetric spreading drop over initially dry thin porous substrate with thickness $\Delta .1$ - liquid drop; 2 - wetted region inside the porous substrate; 3 - dry region inside the porous substrate; $L(t)$ - radius of the drop base; $I(t)$ - radius of the wetted area inside the porous substrate; $r, z$ - co-ordinate system; $h(t, r)$ - profile of the spreading drop.

Spreading of small liquid drops over thin porous layers saturated with the same liquid was investigated in [37]. It was shown that spreading is governed by the same power law as in the case of spreading over a dry solid substrate. The Brinkman's equations were used to model the liquid flow inside the porous substrate. An equation of the drop spreading was deduced, which shows that both an effective lubrication and the liquid exchange between the drop and the porous substrates are equally important. The presence of these two phenomena removes the well-known singularity at the moving three-phase contact line. Matching of the drop profile in the vicinity of the three-phase contact line with the main spherical part of the drop gives the possibility to calculate the pre-exponential factor in the spreading law via permeability and effective viscosity of the liquid in the porous layer. Unfortunately, the latter 
dependency turns out to be very weak and did not allow extracting the effective viscosity of liquids inside porous substrates.

In the case of spreading over dry thin porous layer the drop motion over a porous layer is caused by an interplay of two processes: (a) the spreading of the drop over already saturated parts of the porous layer, which results in an expanding of the drop base, (b) the imbibition of the liquid from the drop into the porous substrate, which results in a shrinkage of the drop base and an expanding of the wetted region inside the porous layer. As a result of these two competing processes the radius of the drop goes through a maximum value over time. A system of two differential equations has been derived to describe the evolution with time of radii of both the drop base and the wetted region inside the porous layer [38]. This system includes two parameters, one account for the effective lubrication coefficient of the liquid over the wetted porous substrate, and the other is a combination of permeability and effective capillary pressure inside the porous layer. The effective lubrication coefficient was determined earlier [37]. An additional experiment has been arranged for an independent determination of the second parameter. After that the system of differential equations does not include any fitting parameters. Experiments were carried out on the spreading of silicone oil drops over various dry microfiltration membranes (permeable in both normal and tangential directions) [38]. The time evolution of the radii of both the drop base and the wetted region inside the porous layer were monitored. All experimental data fell on two universal curves if appropriate scales were used with a plot of the dimensionless radii of the drop base and of the wetted region inside the porous layer on dimensionless time. The predicted theoretical relationships are two universal curves accounting quite satisfactory for the experimental data. According to the theory predictions [38] (i) the dynamic contact angle dependence on the same dimensionless time as before should be a universal function; (ii) the dynamic contact angle should change rapidly over an initial short stage of spreading and should remain a constant value over the duration of the rest of the spreading process. The constancy of the contact angle on this stage has nothing to do with hysteresis of the contact angle: there is no hysteresis in our system. These conclusions again are in the good agreement with experimental observations [38] (Figs. 14, 15).

Kinetics of spreading of surfactant solutions over thick porous substrates has been investigated experimentally in the case of complete wetting [39] as well as 
spreading and simultaneous imbibitions of surfactant solutions [40-41]. These two areas of research are still far from being complete.

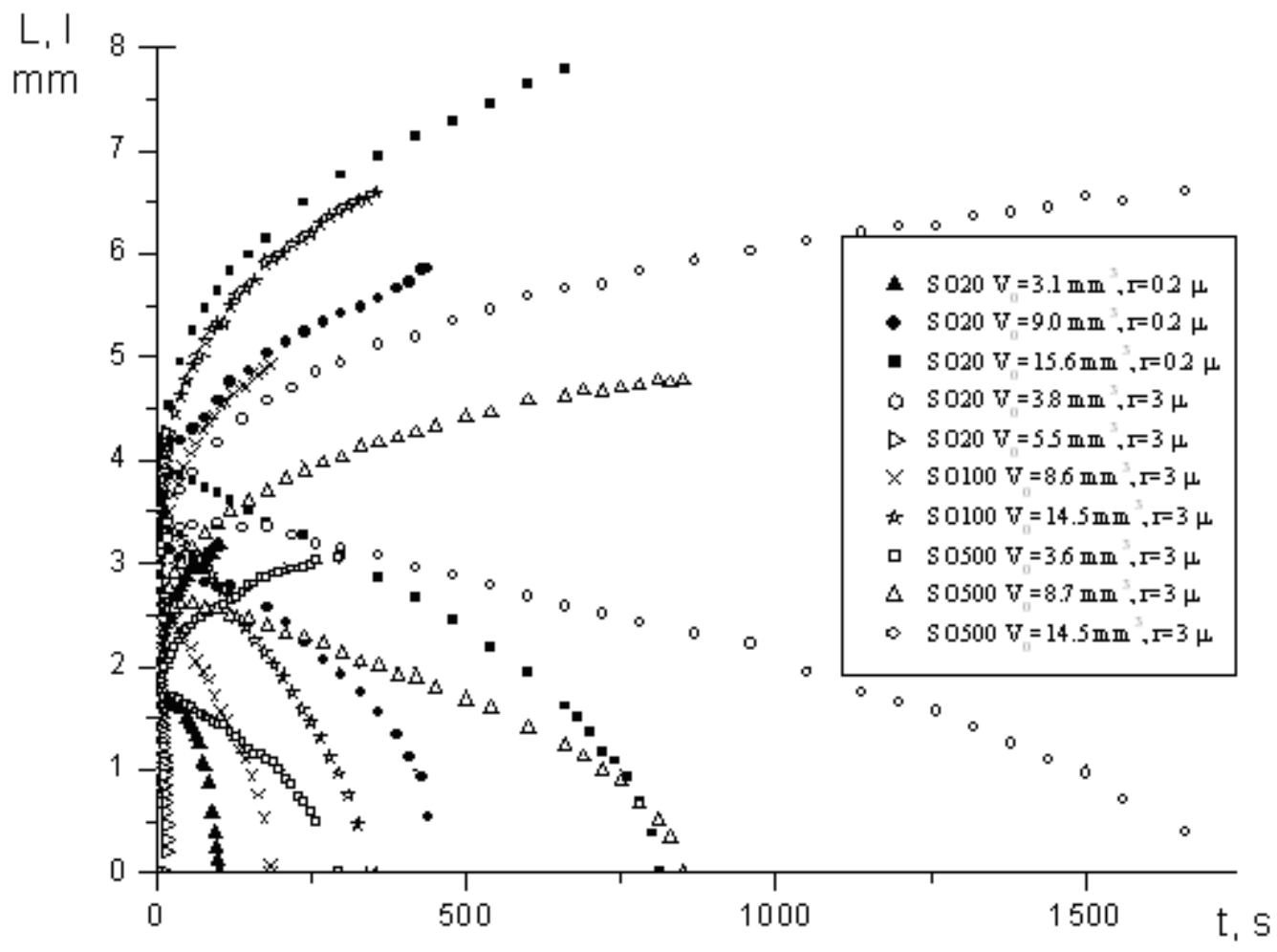

Fig. 14. Measured dependencies of radii of the silicone oil (wide range of viscosities) drops base $(L, \mathrm{~mm})$ and radii of the wetted region inside the porous substrate $(I, \mathrm{~mm})$ on time $(t, s)$. Porous substrates are nitrocellulose membranes of various average pore size $r$. 


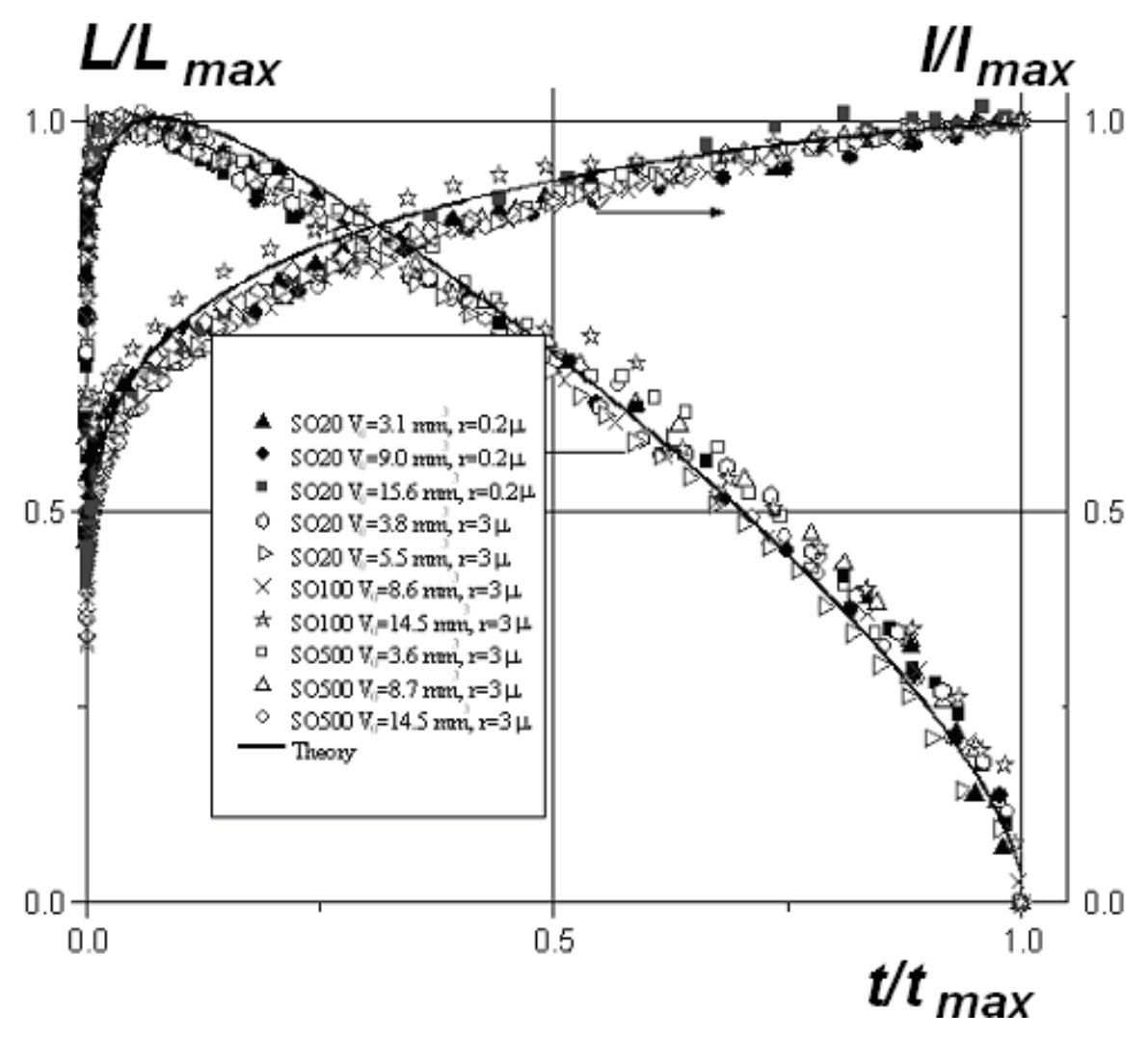

Fig. 15. The same as in Fig. 14 but using dimensionless co-ordinates: $L / L_{\max }, \quad l / l_{\max }, \quad t / t_{\max }$, where $L_{\max }$ is the maximum value of the drop base, which is reached at the moment $t_{m}$. Solid lines according to the deduced theoretical equations. Note, the theory does not include any fitting parameters!

\section{Simultaneous spreading and evaporation}

Over last decades kinetics of spreading and simultaneous evaporation has drawn a considerable attention [11-12, 42-46]. It was experimentally discovered that the rate of evaporation is proportional not the area of the droplet but to the perimeter of the spreading droplet [11, 42-44] (Fig. 16). After that numerous theoretical and experimental efforts were invested in that area. However, the evaporation flux has not been calculated in a self-consistent way [45]: the latent heat of evaporation was not taken into account. The first attempt to calculate the evaporation flux taken into account the latent heat of evaporation in a self-consistent way was undertaken in [12]. The self-consistent way means that the evaporation flux was calculated simultaneously with the calculation of the temperature distribution inside the evaporating droplet. The cooling induced by evaporation results in a non-uniform 
temperature profile along the interface, being colder on the apex and hotter near the contact line [46]. The first attempt to give a physical explanation of the peculiar behaviour of the evaporation flux (Fig.16) was given in [12]. However, in [12] a thermocapillary flow was not taken into account. Interfacial temperature gradient [46] leads to Marangoni thermocpaillary driven flow within the drop.

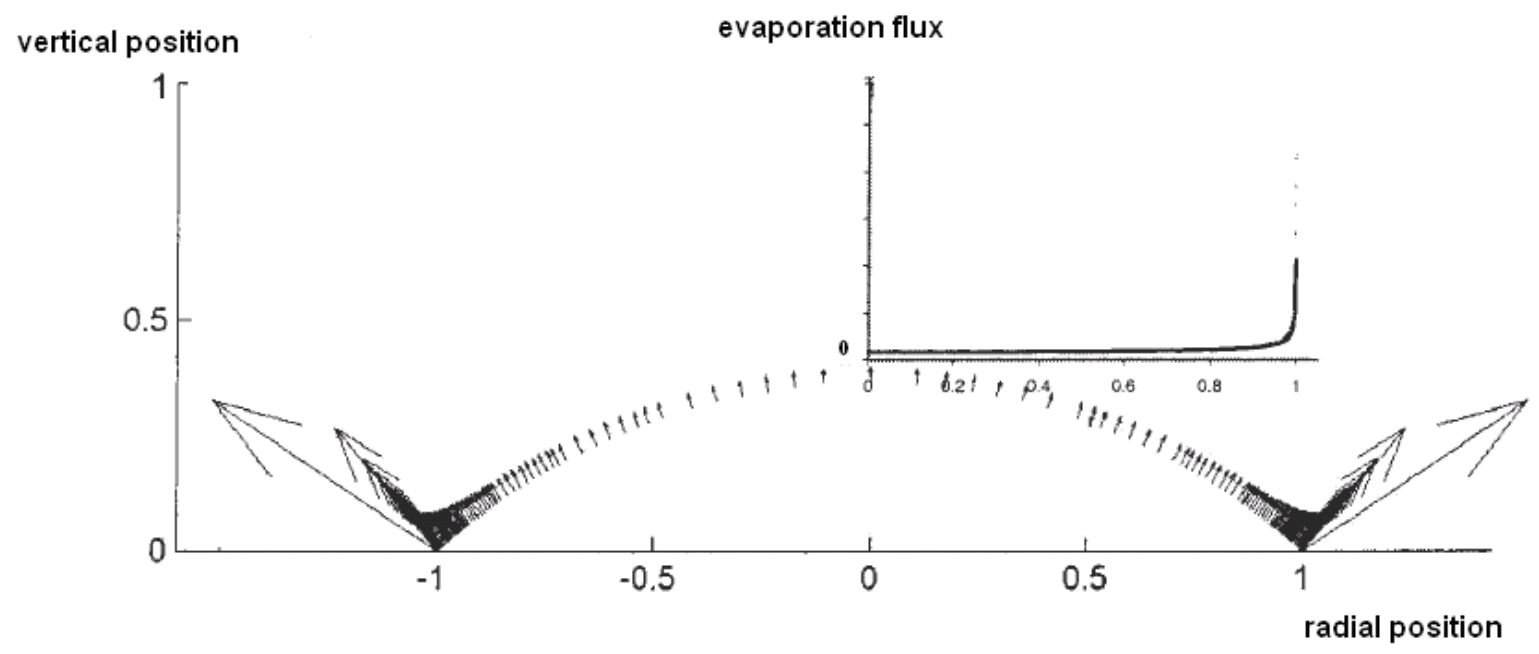

Fig. 18. Evaporation flux along a sessile drop surface [46].

The presence of thin layers at the edge of evaporating droplets and their influence on the kinetics of evaporation is proven to be of a crucial importance for the kinetics of evaporation [12]. However, the influence of surface tension gradient and convection inside evaporating droplets is to be investigated in a selfconsistent way. Experiments on spreading and evaporation of sessile droplets on a solid substrate under various conditions are reported and compared to the developed theoretical model [47]. The liquids used were alkanes: heptane and octane. All liquids completely wet glass substrates used. The time evolution of the radius of the droplet base, contact angle and the droplet height were monitored. The developed theoretical model predicts that measured radius and contact angle data, and the subsequently calculated volume data, would fall onto respective theoretical 'universal curves'. Experimental data both extracted from literature and the authors' own confirmed this theoretical prediction. The predicted universal curves fairly fit experimental data (Fig. 17). 


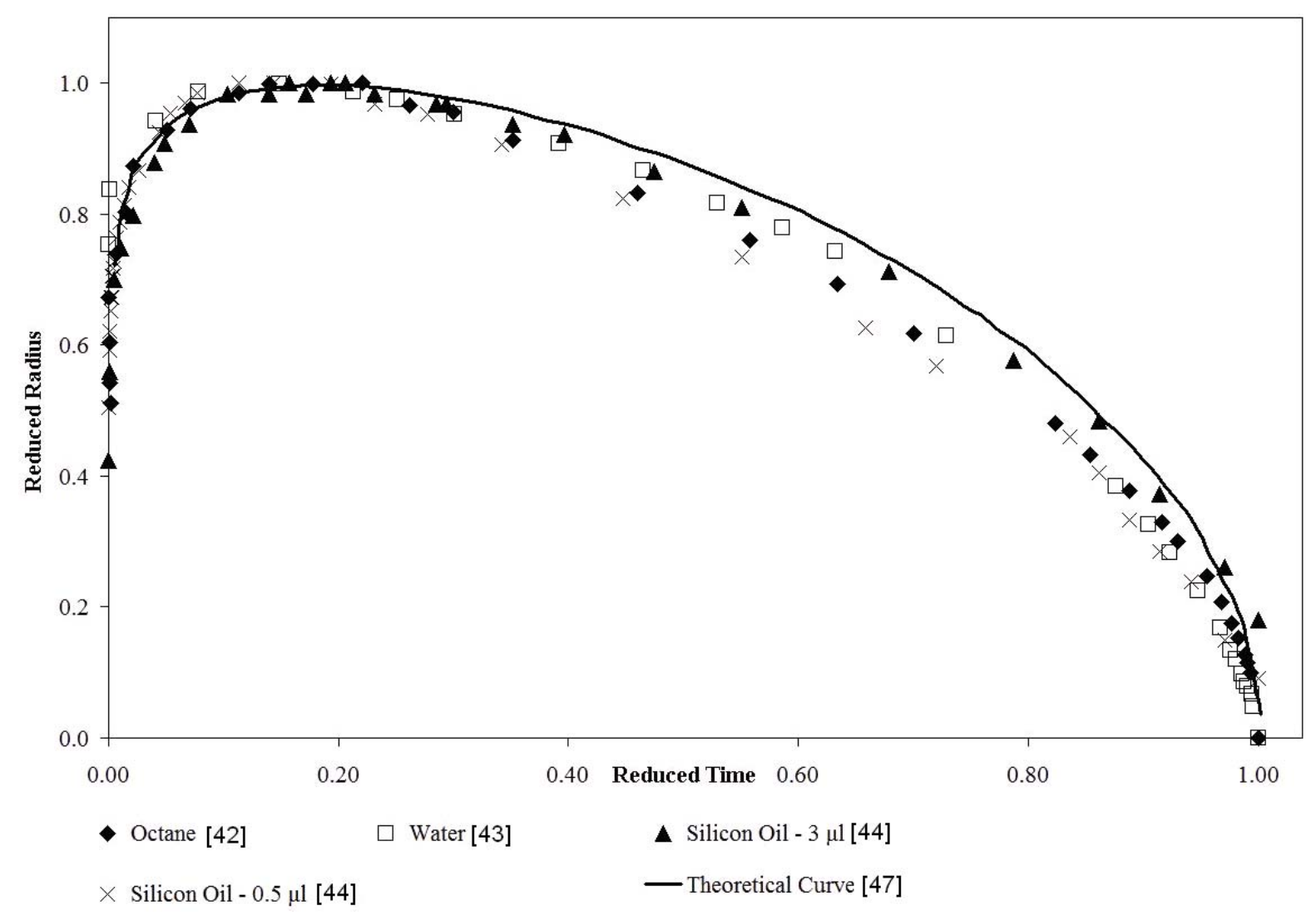

Fig. 17. A dimensionless radius against dimensionless time curve for the behaviour of the droplet radius comparing different liquids spreading/evaporating on solid substrates extracted from literature and theoretical prediction. The solid line is calculated according to the developed theory.

Surfactant solutions are used in agriculture to deliver aqueous droplets on hydrophobic plat lives. Immediately after deposition the droplets start to spread out and evaporate. That is, kinetics of spreading and evaporation of surfactant solutions is one of very important problems in the area. It was mentioned above that experimental investigation started only recently [13] and since then the area attracts a considerable attention ever since [48]. Kinetics of spreading and simultaneous evaporation has been investigated experimentally in the case of mixture of two liquids [49]; however a theoretical understanding in this case is to be developed.

6. Spreading of aqueous droplets and aqueous surfactant solutions 
Spreading of aqueous drops induced by the overturning of amphiphilic molecules or their fragments in the surface layer of an initially hydrophobic substrate.

The spontaneous spreading of aqueous droplet on initially hydrophobic solid surfaces built up by amphiphilic molecules, which are immobile in a tangential direction but can rotate in a vertical direction, was discovered in [50]. Rotation of amphiphilic molecules (or their amphiphilic fragments) of the substrate surface layer creates hydrophilic parts on the surface and promote the spreading as a result.

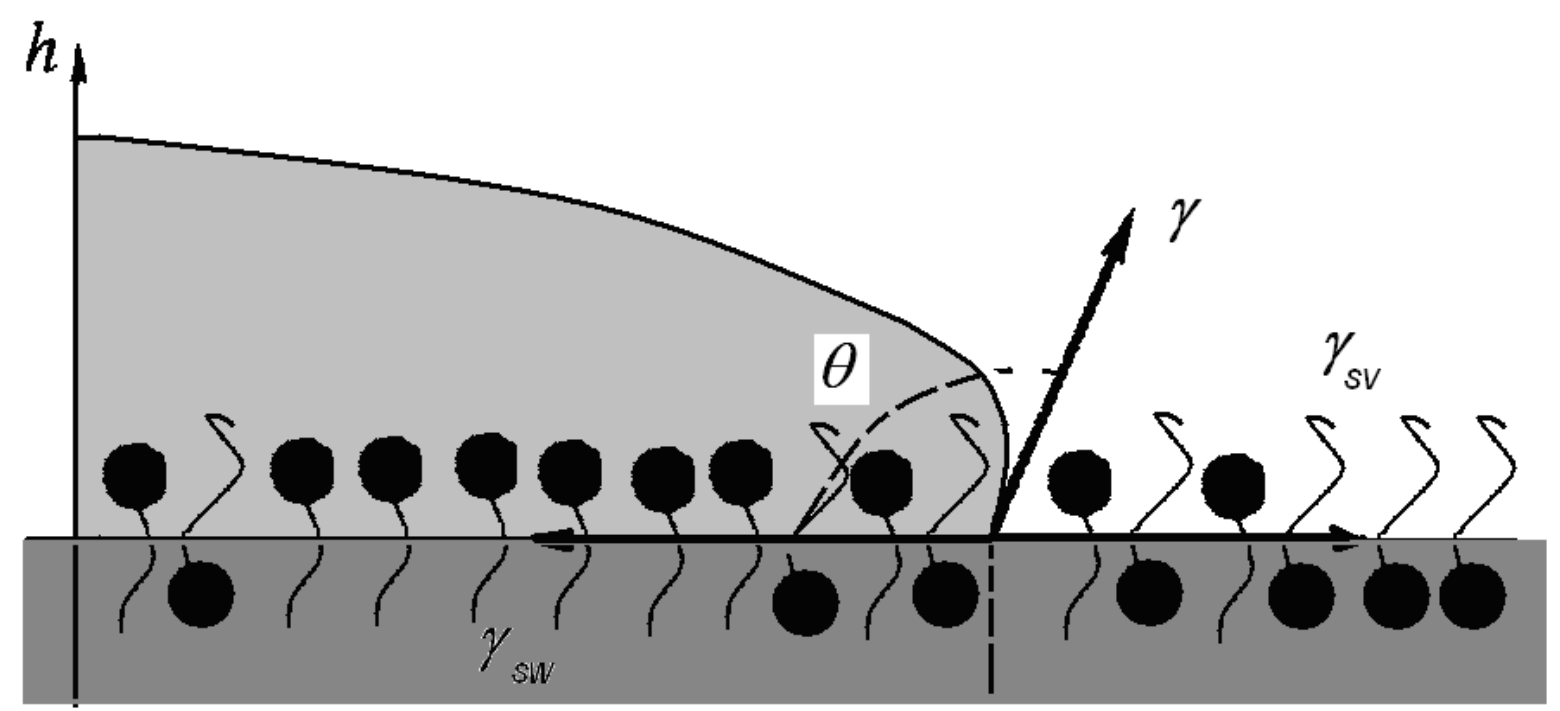

Fig. 18. Partial hydrophilisation of the initially hydrophobic polymer substrate determined by an overturning of amphiphilic fragments of polymer in front of the moving three phase contact line.

Such a situation may occur, for example, during the contact of an aqueous drop with the surface of a polymer whose macromolecules have amphiphilic surface groups capable of rotating around the backbone or during the wetting of polymers containing surface-active additives or Langmuir-Blodgett films composed of amphiphilic molecules.

However, the process is more sophisticated than it looks: it is shown in [51] that this phenomenon is similar to autophilic phenomenon. It has been shown [51] that drop spreading is possible only if there is lateral interaction between neighboring amphiphilic molecules (or groups). This interaction leads to a tangential transfer of the "overturned state" to some distance ahead of the advancing three-phase contact 
line making it partially hydrophilic (Fig. 18). Depending on the structure of the polymer (macro)molecules, the physical state of the polymer, and other factors, such a rearrangement may involve various forms of molecular motion, from the reorientation of individual amphiphilic lateral groups (when their rotation around the backbone is allowed) to the diffusion of the macromolecules as a whole (autophilic phenomenon). Each of these processes may occur on different time scales. If the characteristic time scale of self-organization is comparable with the time of measurement, this process may be observed while studying the time evolution of the contact angle (and size) of an aqueous drop over a polymer. Langmuir was the first to mention the possible reorientation ("overturning") of amphiphilic molecules in contact with a polar liquid. Experiments have illustrated such process in mono layers and Langmuir-Blodgett films composed of long-chain fatty acids and at the surface of mixtures of such acids with paraffin in contact with an aqueous drop, resulting in gradual drop spreading [50]. Such situation has also been observed during the spreading of drops over polymeric surfaces, when the amphiphilic groups of their macromolecules are capable of reorientation by rotating around the macromolecule backbone [50]. A theory describing the kinetics of droplet spreading has been developed with allowance for this mechanism of self-organization of the surface layer of a substrate in the contact with a droplet [51].

Spreading of surfactants over thin viscous liquid layers.

A moving circular wave front forms after a small droplet of aqueous surfactant solution is deposited on a thin aqueous layer (Fig. 19). The time evolution of the radius of the moving front can be monitored and information on the properties of surfactants can be extracted [52-55]. In [53] surfactants of different solubility were used at concentrations above CMC. It is shown that the time evolution of the moving front proceeds in two stages: a rapid first stage, which is followed by a slower second stage. It has been shown that the time evolution of the moving front substantially depends on the surfactant solubility. An exact solution for the evolution of the moving front has been deduced for the case of low soluble surfactants. A qualitative theory has been developed to account for the influence of the solubility on the front motion. Experimental observations [53] are in a good agreement with the theory predictions. 


\section{a}

\begin{tabular}{|ll|}
\hline water layer $\quad \mathbf{h}_{0}$ \\
\hline
\end{tabular}

\section{b}

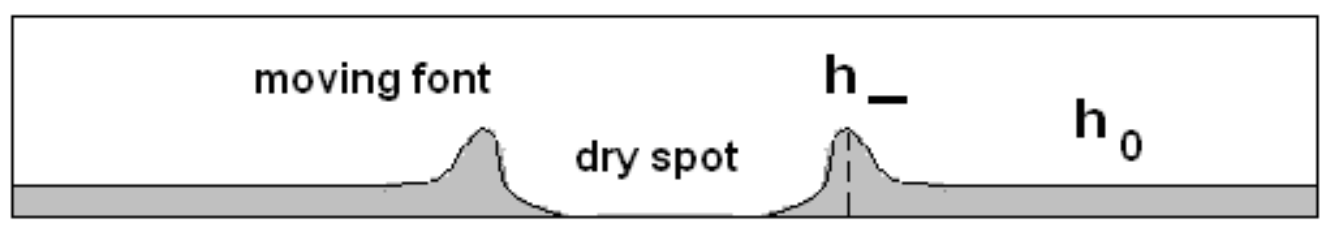

$r(t) \quad R(t)$

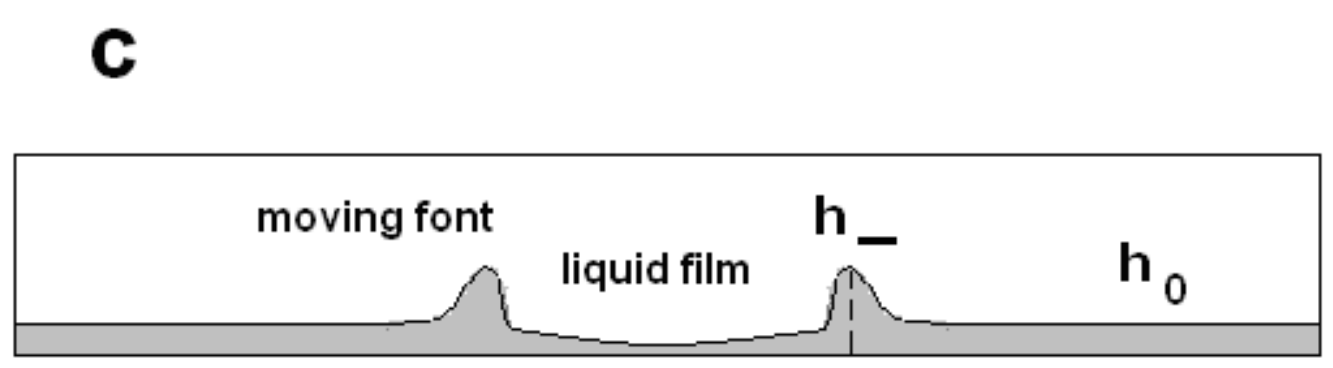

$\mathbf{R}(\mathbf{t})$

Fig. 19. a - a small droplet of aqueous surfactant solution is deposited on a top of thin aqueous layer of thickness $h_{0} ; b$ - dry spot formation in the centre: cross section of the system: $R(t)$ - radius of a circular moving front, $r(t)$ - radius of dry spot in the centre, $H$ - the height of the moving front; $c$ - the same as in the previous case (b) without dry spot formation in the centre.

The identical experimental arrangement can be used for investigation of behaviour of trisiloxane solutions ("superspreaders") [55]. It is shown that the time evolution of the moving front in the case of trisiloxane solutions differs substantially from that of regular surfactants. 


\section{Acknowledgement}

This research was supported by the Engineering and Physical Sciences Research Council, UK, and by EU under Grant MULTIFLOW, FP7-ITN- 2008214919.

\section{References}

1. Young T. Philos. Trans R Soc London 1805; 95: 65.

2. Cassie ABD, Baxter S. Trans Faraday Soc 1944; 40: 546.

3. Wenzel RN. Ind Eng Chem 1936; 28: 998.

4. Deryaguin BV, Churaev NV, Muller VM: Surface forces. Consultants Bureau, Plenum Press, New York; 1987.

5. Israelachvili JN: Intermolecular and surface forces. 2nd ed., Academic Press, London; 1991.

6. Russel WB, Saville DA, Schowalter WR: Colloidal Dispersions. Cambridge University Press; 1999.

7. Exerowa D, Kruglyakov P: Foam and foam films: theory, experiment, application. Elsevier, New York; 1998.

8. Starov VM, Velarde MG. J Physics: Condenced Matter 2009; 21: 464121.

9. Starov VM, Velarde MG, Radke CJ: Dynamics of wetting and spreading. Taylor\&Frances, in "Surfactant Sciences Series", 138; 2007.

10. Gokhale ShJ, Plawsky JL, Wayner Jr PC. Adv Colloid Interface Sci 2003; 104: 175.

11. Gu'ena G, Allancon P, Cazabat AM. Colloids and Surfaces A: Physicochem Eng Aspects 2007; 300: 307.

12. Moosman S, Homsy GM. J Colloid Interface Sci 1979; 73 (1): 212.

13. Soboleva OA, Summ B D. Colloid J, Russian |Academy of Sciences, English Translation 2003; 65(1): 89.

14. Starov VM, Adv Colloid Interface Sci 2004; 111: 3.

15. Chengara A, Nikolov AD, Wasan DT, Trokhymchuk A, Henderson D. J Colloid Interface Science 2004; 280: 192.

16. Miller CA, Ruckenstein E. J Colloid Interface Sci 1974; 48(3): 368. 
17. Churaev NV. Adv Colloid Interface Sci 2003; 103: 197.

18. Hughes M: Physical Chemistry. Pergamon Press; 1961.

19. Alexandrova AD, Toshev BV, Scheludko AD. Colloids and Surfaces A: Physicochemical and Engineering Aspects1993; 79: 43.

20. Holdich R, Starov VM, Prokopovich P, Njobuenwu DO, Rubio RG, Zhdanov S, Velarde MG. Colloids and Surfaces A: Physicochemical and Engineering Aspects 2006; 282-283: 247.

21. Platikanov D, Nedyalkov M, Petkova V. Adv Colloid Interface Sci 2003; 100-102: 185.

22. Petkova V, Platikanov D, Nedyalkov M, Adv Colloid Interface Sci 2003; 104: 37.

23. Zorin ZM, Churaev NV, Cololid J., USSR Academy of Sciences, 1968; 30: 371 (in Russian).

24. Deryagin BV, Ershova IG, Churaev NV, Dokl. Akad. Nauk SSSR, 1968; 182: 368 (in Russian).

25. Viktorina MM, Deryagin BV, Ershova IG, Churaev NV, Dokl. Akad. Nauk SSSR, 1971; 200: 1306 (in Russian).

26. Mack GL. J Phys Chem, 1936; 40 (2): 159.

27. Veselovsky VS, Pertsev VN, J Phys Chem (USSR Academy of Sciences), 1936; 8 (2): 245 (in Russian).

28. Blake TD. J Colloid Interface Sci 2006; 299: 1

29. Blake TD, Haynes JM. J Colloid Interface Sci 1969; 30: 421.

30. Blake TD, De Coninck J. Adv Colloid Interface Sci 2002; 96: 21.

31. de Ruijter M J, De Coninck J, Oshanin G. Langmuir 1999; 15: 2209.

32. Decamps C, De Coninck J. Langmuir 2000; 16: 10150.

33. Ruckenstein E, Dunn CS. J Colloid Interface Sci 1977; 59(1): 135.

34. Starov VM, Kalinin VV, Chen J-D. Adv Colloid Interface Sci1994; 50: 187.

35. Daniel RC, Berg JC. Adv Colloid Interface Sci 2006; 123-126: 439.

36. Starov VM, Zhdanov SA, Velarde MG, Kosvintsev SR, Sobolev VD. Adv Colloid Interface Sci 2003; 104: 123.

37. Starov VM, Kosvintsev SR, Sobolev VD, Velarde MG, Zhdanov SA. J Colloid Interface Sci 2002; 246(2): 372. 
38. Starov VM, Kosvintsev SR, Sobolev VD, Velarde MG, Zhdanov SA. J Colloid Interface Sci 2002; 252: 397.

39. Starov VM, Zhdanov SA, Velarde MG. Langmiur 2002; 18 (25): 9744.

40. Zhdanov SA, Starov VM, Sobolev VD, Velarde MG. J Colloid Interface Sci 2003; 264: 481.

41. Starov VM, Zhdanov SA, Velarde MG. J Colloid Interface Sci 2004; 273 (2): 589.

42. Guéna G, Poulard C, Vou'e M, De Coninck J, Cazabat A-M. Colloids and Surfaces A: Physicochemical and Engineering Aspects 2006; $291: 191$.

43. Guéna G, Poulard C, Cazabat A-M. Colloids and Surfaces A: Physicochemical and Engineering Aspects 2007; 298: 2.

44. Guéna G, Poulard C, Cazabat A-M. J Colloid Interface Sci 2007; 312 (1): 164.

45. Deegan RD, Bakajin O, Dupont TF, Huber G, Nagel SR, Witten ThA. Phys Rev E 2000; 62(1); 756.

46. Starov V, Sefiane K. Colloids and Surfaces A: Physicochemical and Engineering Aspects 2009; 333 (1-3):170.

47.Lee KS, Cheah CY, Copleston RJ, Starov VM, Sefiane K. Colloids and Surfaces A: Physicochemical and Engineering Aspects 2007; 323 (1-3): 63.

48. Sefiane K. J Petroleum Sci Engineering 2006; 51: 238.

49. David S. "An Investigation of the Wetting Behaviour of Evaporating Drops". PhD Thesis, University of Edinburgh, UK, 2008.

50. Rideal E, Tadayon J. Proc Roy Soc (London) A, 1954; 225(1162): 346.

51. Starov VM, Rudoy VM, Ivanov VI. Colloid J (Russian Academy of Scinces, English translation) 1999; 61 (3): 374.

52. Starov VM, de Ryck A, Velarde MG. J Colloid Interface Sci 1997; 190 (1): 104.

53. Lee KS, Starov VM. J Colloid Interface Sci 2007; 314: 631.

54. Lee KS, Starov VM. J Colloid Interface Sci 2009; 329 (2): 361.

55. Lee KS, Starov VM, Muchatuta TJP, Srikantha SIR. Colloid J Russian Academy of Sci, English Translation 2009; 71(3): 368. 